\title{
Orbit and Dynamical Mass of the Late-T Dwarf GL 758 B $^{*}$
}

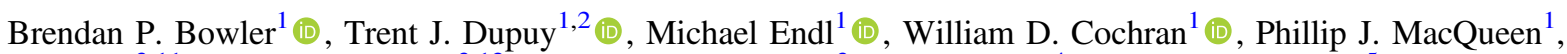 \\ Benjamin J. Fulton ${ }^{3,11}$ (D), Erik A. Petigura ${ }^{3,12}$ (D) , Andrew W. Howard $^{3}$ (D), Lea Hirsch ${ }^{4}$ (D), Kaitlin M. Kratter ${ }^{5}$ (D) , Justin R. Crepp ${ }^{6}$ (D), \\ Beth A. Biller ${ }^{7}$ (D), Marshall C. Johnson ${ }^{8}$ (D), and Robert A. Wittenmyer ${ }^{9,10}$ (D) \\ ${ }^{1}$ McDonald Observatory and the Department of Astronomy, The University of Texas at Austin, Austin, TX 78712, USA; bpbowler@astro.as.utexas.edu \\ ${ }^{2}$ Gemini Observatory, Northern Operations Center, 670 N. Aohoku Place, Hilo, HI 96720, USA \\ ${ }^{3}$ California Institute of Technology, 1200 E. California Blvd., Pasadena, CA 91125, USA \\ ${ }^{4}$ Astronomy Department, University of California, Berkeley CA 94720-3411, USA \\ 5 Department of Astronomy, University of Arizona, Tucson, AZ 85721, USA \\ ${ }^{6}$ Department of Physics, University of Notre Dame, 225 Nieuwland Science Hall, Notre Dame, IN 46556, USA \\ ${ }^{7}$ Institute for Astronomy, University of Edinburgh, Blackford Hill View, Edinburgh EH9 3HJ, UK \\ ${ }^{8}$ Department of Astronomy, The Ohio State University, 140 West 18th Avenue, Columbus, OH 43212 USA \\ ${ }^{9}$ University of Southern Queensland, Computational Engineering and Science Research Centre, Toowoomba, Queensland 4350, Australia \\ ${ }^{10}$ Australian Centre for Astrobiology, University of New South Wales, Sydney, NSW 2052, Australia \\ Received 2018 January 1; revised 2018 February 21; accepted 2018 February 23; published 2018 March 21
}

\begin{abstract}
Gl 758 B is a late-T dwarf orbiting a metal-rich Sun-like star at a projected separation of $\rho \approx 1$ !"6 (25 au). We present four epochs of astrometry of this system with NIRC2 at Keck Observatory spanning 2010 to 2017 together with 630 radial velocities (RVs) of the host star acquired over the past two decades from McDonald Observatory, Keck Observatory, and the Automated Planet Finder at Lick Observatory. The RVs reveal that Gl 758 is accelerating with an evolving rate that varies between 2 and $5 \mathrm{~m} \mathrm{~s}^{-1} \mathrm{yr}^{-1}$, consistent with the expected influence of the imaged companion Gl $758 \mathrm{~B}$. A joint fit of the RVs and astrometry yields a dynamical mass of $42_{-7}^{+19} M_{\text {Jup }}$ for the companion with a robust lower limit of $30.5 M_{\text {Jup }}$ at the $4-\sigma$ level. Gl $758 \mathrm{~B}$ is on an eccentric orbit ( $e=0.26-0.67$ at $95 \%$ confidence) with a semimajor axis of $a=21.1_{-1.3}^{+2.7}$ au and an orbital period of $P=96_{-9}^{+21} \mathrm{yr}$, which takes it within $\approx 9$ au from its host star at periastron passage. Substellar evolutionary models generally underpredict the mass of G1 758 B for nominal ages of 1-6 Gyr that have previously been adopted for the host star. This discrepancy can be reconciled if the system is older-which is consistent with activity indicators and recent isochrone fitting of the host star-or alternatively if the models are systematically overluminous by $\approx 0.1-0.2$ dex. Gl 758 B is currently the lowest-mass directly imaged companion inducing a measured acceleration on its host star. In the future, bridging RVs and high-contrast imaging with the next generation of extremely large telescopes and space-based facilities will open the door to the first dynamical mass measurements of imaged exoplanets.
\end{abstract}

Key words: brown dwarfs - stars: individual (Gl 758)

Supporting material: machine-readable table

\section{Introduction}

Brown dwarfs and giant planets radiatively cool over time and follow mass-luminosity-age relationships. Two quantitiesusually luminosity and age-are needed to infer the third using substellar evolutionary models, which is how the masses of all directly imaged exoplanets and the vast majority of brown dwarfs are estimated. Fundamental tests of these cooling models require measurements of all three parameters, making model-independent dynamical masses especially valuable for the subset of brown dwarfs with well-constrained ages. These rare benchmarks with measured luminosities, ages, and dynamical masses have shown that widely used hot-start evolutionary models systematically over-predict masses by up to $25 \%$, a discrepancy that may originate from diverse accretion histories or incomplete modeling of cloud evolution from $\mathrm{L}$ to $\mathrm{T}$ spectral classes (Dupuy et al. 2009, 2014; Crepp et al. 2012), although the exact source

\footnotetext{
* Some of the data presented herein were obtained at the W.M. Keck Observatory, which is operated as a scientific partnership among the California Institute of Technology, the University of California and the National Aeronautics and Space Administration. The Observatory was made possible by the generous financial support of the W.M. Keck Foundation.

11 Texaco Fellow.

12 Hubble Fellow.
}

of this deviation remains unclear. Expanding these tests to even lower brown dwarf masses - and ultimately into the planetary regime-will enable precision tests of giant planet evolutionary, atmospheric, and formation models.

An especially useful class of benchmark brown dwarfs are those orbiting at wide enough separations to be identified and characterized with direct imaging, but close enough that their influence on their host stars can be readily measured with radial velocities (RVs). One of the legacy products of long-baseline precision RV planet searches operating over the past 20 years is the identification of systems exhibiting shallow accelerations. These "dynamical beacons" point to distant stellar, substellar, or planetary companions and are excellent targets for follow-up high-contrast imaging to determine the nature of the perturbing body (e.g., Bowler 2016).

Four high-mass brown dwarfs have been recovered with high-contrast imaging based on long-term RV trends from their host stars: HR 7672 B (Liu et al. 2002), HD 19467 B (Crepp et al. 2014), HD 4747 B (Crepp et al. 2016), and HD 4113 C (Cheetham et al. 2017). These benchmark brown dwarfs have mid-L to late-T spectral types, dynamical masses between 50 and $70 M_{\mathrm{Jup}}$, and ages between 1 and $7 \mathrm{Gyr}$. HD $4113 \mathrm{C}$ is especially peculiar; the inferred mass of this late- $\mathrm{T}$ dwarf companion is about a factor of two lower than its dynamical 
mass, suggesting that it may be an unresolved binary T dwarf. In addition, over two dozen brown dwarfs in close binaries have had their masses measured through patient orbit monitoring campaigns (e.g., Liu et al. 2008; Konopacky et al. 2010; Dupuy \& Liu 2017). However, their ages are usually difficult to independently constrain, even with component-resolved spectroscopy, unless these binaries also happen to be gravitationally bound to a host star (Potter et al. 2002; McCaughrean et al. 2004; Ireland et al. 2008; Dupuy et al. 2009).

In this work, we present a dynamical mass measurement of the late-T dwarf Gl 758 B based on new high-contrast imaging observations from Keck/NIRC2 together with $630 \mathrm{RVs}$ of the host star from McDonald Observatory, Keck Observatory, and the Automated Planet Finder (APF) gathered over the past 20 years. Orbital motion is evident in all data sets; Gl 758 B displays changes in position angle (P.A.) and separation in our imaging data, and our precision RVs show clear signs of a shallow acceleration with slight curvature. With a dynamical mass of $42_{-7}^{+19} M_{\text {Jup }}$, Gl $758 \mathrm{~B}$ is likely to be the lowest-mass imaged companion inducing a measured acceleration on its host star and serves as a valuable test for substellar evolutionary models.

Section 2 provides an overview of the Gl 758 system and summarizes the physical properties of the late-T dwarf companion. Section 3 describes the precision RV observations of the host star. Our Keck observations, point-spread function (PSF) subtraction, and astrometric measurements are reported in Section 4. The joint Keplerian fit to the RV and astrometric data can be found in Section 5. Finally, we compare the results to predictions from evolutionary models in Section 6 and conclude in Section 7.

\section{Overview of the Gl 758 System}

Gl 758 (=HD182488, HR 7368) is a bright $(V=6.3 \mathrm{mag})$ G8 star located at $15.66 \pm 0.09$ pc (Gaia Collaboration et al. 2016). Activity, lithium, and kinematics of this star all point to an age of $3_{-2}^{+3} \mathrm{Gyr}$, implying a mass of about $0.97 \mathrm{M}_{\odot}$, and enhanced metallicity of $[\mathrm{Fe} / \mathrm{H}] \approx+0.2 \mathrm{dex}$ (see Vigan et al. 2016 for a thorough summary).

The brown dwarf companion Gl 758 B was first discovered by Thalmann et al. (2009) as part of the SEEDS high-contrast imaging survey (Tamura 2016). Further photometric and spectroscopic characterization by Currie et al. (2010), Janson et al. (2011), Vigan et al. (2016), and Nilsson et al. (2017) established it as a late-T dwarf (T7-T8) with a model-based mass between 10 and $40 M_{\text {Jup }}$ and an effective temperature of 600-750 K. Vigan et al. (2016) find that no empirical or model template accurately reproduces the ensemble of photometry for this object, possibly due to an enhanced metallicity. Although Gl 758 B has only completed a small fraction of its orbit, the most likely orbital solutions relying solely on astrometry suggest it is eccentric with a semimajor axis between about 20 and $60 \mathrm{au}$. Nilsson et al. (2017) propose that the acceleration induced by Gl $758 \mathrm{~B}$ should be measurable on the host star, but no evidence of a trend was observed by Vigan et al. (2016) using RVs from the ELODIE spectrograph and Lick Observatory, most likely due to the relatively large uncertainties of these data sets.

\section{Radial Velocity Observations}

\subsection{Harlan J. Smith Telescope/Tull Spectrograph}

Gl 758 was included in the target sample of the longduration RV planet search at McDonald Observatory (e.g.,
Table 1

Relative Radial Velocities

\begin{tabular}{|c|c|c|c|}
\hline $\begin{array}{l}\text { Date } \\
\left(\mathrm{BJD}_{\mathrm{TDB}}\right)\end{array}$ & $\begin{array}{c}\mathrm{RV} \\
\left(\mathrm{m} \mathrm{s}^{-1}\right)\end{array}$ & $\left(\begin{array}{c}\sigma_{\mathrm{RV}} \\
\left(\mathrm{m} \mathrm{s}^{-1}\right)\end{array}\right.$ & Obs. \\
\hline \multicolumn{4}{|c|}{ McDonald Observatory } \\
\hline 2451121.66132 & 29.88 & 4.63 & $\mathrm{McD}$ \\
\hline 2451152.55410 & 20.29 & 4.57 & $\mathrm{McD}$ \\
\hline 2451328.93255 & 18.48 & 3.78 & $\mathrm{McD}$ \\
\hline 2451360.93134 & 17.64 & 4.02 & $\mathrm{McD}$ \\
\hline 2451417.85387 & 13.14 & 4.49 & $\mathrm{McD}$ \\
\hline 2451452.68967 & 12.89 & 4.15 & $\mathrm{McD}$ \\
\hline 2451503.57734 & 21.52 & 4.73 & $\mathrm{McD}$ \\
\hline 2451530.54249 & 13.60 & 4.10 & $\mathrm{McD}$ \\
\hline 2451686.95063 & 5.80 & 3.57 & $\mathrm{McD}$ \\
\hline 2451751.77696 & 13.70 & 3.98 & $\mathrm{McD}$ \\
\hline \multicolumn{4}{|c|}{ Keck Observatory } \\
\hline 2453927.88034 & 19.41 & 0.99 & Keck \\
\hline 2453927.88136 & 18.40 & 1.03 & Keck \\
\hline 2453927.88237 & 17.27 & 1.13 & Keck \\
\hline 2453982.88766 & 17.25 & 0.90 & Keck \\
\hline 2453982.88864 & 14.97 & 0.96 & Keck \\
\hline 2453982.88965 & 15.15 & 0.95 & Keck \\
\hline 2454338.96476 & 14.86 & 1.14 & Keck \\
\hline 2454642.00375 & 11.02 & 1.22 & Keck \\
\hline 2454689.89631 & 10.76 & 1.15 & Keck \\
\hline 2454717.84714 & 5.36 & 1.22 & Keck \\
\hline \multicolumn{4}{|c|}{ APF } \\
\hline 2456505.87953 & 6.17 & 0.89 & $\mathrm{APF}$ \\
\hline 2456506.79254 & 5.16 & 0.93 & APF \\
\hline 2456515.81319 & 7.68 & 1.00 & $\mathrm{APF}$ \\
\hline 2456516.85698 & 9.41 & 0.87 & $\mathrm{APF}$ \\
\hline 2456517.75297 & 10.05 & 0.93 & APF \\
\hline 2456518.77451 & 9.55 & 0.89 & $\mathrm{APF}$ \\
\hline 2456525.74145 & 8.28 & 1.04 & $\mathrm{APF}$ \\
\hline 2456526.74485 & 9.92 & 0.87 & APF \\
\hline 2456530.79148 & 8.83 & 1.05 & $\mathrm{APF}$ \\
\hline 2456534.75735 & 12.16 & 0.92 & $\mathrm{APF}$ \\
\hline
\end{tabular}

(This table is available in its entirety in machine-readable form.)

Cochran et al. 1997; Endl et al. 2016). The Tull Coudé spectrograph (Tull et al. 1995) was used at the Harlan J. Smith $2.7 \mathrm{~m}$ telescope in combination with an $\mathrm{I}_{2}$ cell in the light path to obtain precise differential RVs. We commenced observations of G1 758 on 1998 November 4, and have accumulated 118 precise RV measurements over the past 19 years. Beginning in 2009 an exposure meter was used to provide the optimal exposure level and compute precise barycentric corrections. We measure precise RVs from these spectra using our Austral $\mathrm{I}_{2}$ cell data code (Endl et al. 2000). The exposure times of the GJ 758 spectra range from 365 to 1200 s, primarily controlled by atmospheric seeing conditions to reach the desired signal-to-noise ratio $(\mathrm{S} / \mathrm{N})$ of $\sim 300$ per pixel in the $\mathrm{I}_{2}$ bandpass $(500$ to $650 \mathrm{~nm}$ ). The RV data have a total rms of $13 \mathrm{~m} \mathrm{~s}^{-1}$ and a median uncertainty of $4.6 \mathrm{~m} \mathrm{~s}^{-1}$. Our measurements are listed in Table 1 and displayed in Figure 1.

\subsection{Keck/HIRES}

Gl 758 has been continuously monitored with the High Resolution Echelle Spectrometer (HIRES; Vogt et al. 1994) at the Keck I telescope since 2006. This star was initially targeted 


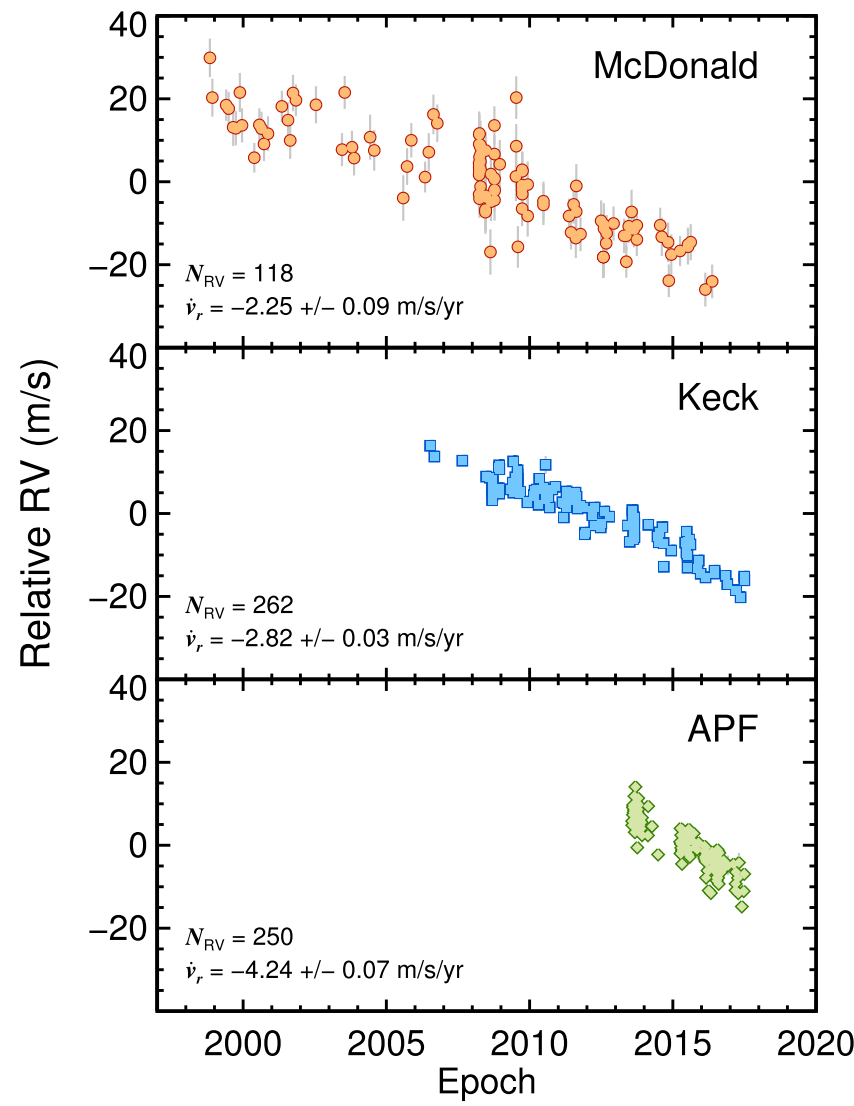

Figure 1. Relative RVs of Gl 758 from the Tull Spectrograph at McDonald Observatory's Harlan J. Smith Telescope (top), HIRES at Keck Observatory (middle), and the Levy Spectrograph at the APF (bottom). A linear fit to each data set shows an evolving and steepening slope at later epochs, listed in the bottom-left corner of each panel, implying recent changes in the radial acceleration of Gl 758 .

as an RV standard for FGK stars in the Kepler field due to its proximity on the sky to the Kepler footprint and its stable RVs, but after several years of coverage it became apparent that Gl 758 was undergoing a shallow radial acceleration (G. Marcy 2016, private communication). Altogether 262 spectra were gathered following the standard setup, observing strategy, and procedure for measuring relative RVs implemented by the California Planet Search program (Howard et al. 2010). An iodine cell is mounted in the optical path before the slit entrance to provide a set of stable reference lines (Marcy \& Butler 1992), an exposure meter is used to consistently achieve a $\mathrm{S} / \mathrm{N}$ of about 225 per reduced pixel near $550 \mathrm{~nm}$, and relative RVs are extracted by forward modeling the stellar and iodine spectra convolved with the instrument line spread function (Valenti et al. 1995). The median uncertainty from these measurements is $1.2 \mathrm{~m} \mathrm{~s}^{-1}$. A secular trend of $-2.82 \pm 0.03 \mathrm{~m} \mathrm{~s}^{-1} \mathrm{yr}^{-1}$ is apparent in our Keck RVs (Figure 1). This slope is slightly steeper than the trend from McDonald Observatory, suggesting a recent change in the acceleration. This is readily apparent by considering only the latest HIRES data, which exhibits a slope of $-3.08 \mathrm{~m} \mathrm{~s}^{-1} \mathrm{yr}^{-1}$ since 2010, and $-4.15 \mathrm{~m} \mathrm{~s}^{-1} \mathrm{yr}^{-1}$ since 2013.5. Our HIRES RVs and uncertainties are listed in Table 1.

\subsection{Automated Planet Finder Telescope/Levy Spectrograph}

Observations of Gl 758 have been carried out autonomously at the $2.4 \mathrm{~m} \mathrm{APF}$ telescope at Lick Observatory since 2013. A total of 250 echelle spectra were gathered as part of APF's automated Doppler search for rocky planets (Fulton et al. 2015) with the Levy Spectrograph, which employs an iodine cell to measure precise relative RVs (Vogt et al. 2014). Each spectrum spans $3740-9700 \AA$ at a resolving power of $\approx 100,000$ with the $1^{\prime \prime}$ decker. RVs are measured by forward-modeling 848 spectral regions, and the resulting variance is adopted as the RV uncertainty. The median instrumental precision of these measurements for Gl 758 is $1.4 \mathrm{~m} \mathrm{~s}^{-1}$. The APF RVs reveal an acceleration that is significantly steeper than the McDonald and Keck RVs (Figure 1). The linear trend from APF is $-4.24 \pm 0.07 \mathrm{~m} \mathrm{~s}^{-1} \mathrm{yr}^{-1}$, indicating a substantial evolution in recent years. Fortunately, these evolving slopes provide curvature that can better constrain the orbit and dynamical mass of the companion. Our APF RVs and uncertainties are listed in Table 1.

\section{Astrometric Observations}

\subsection{Keck/NIRC2 Adaptive Optics Imaging}

We observed Gl 758 with the NIRC2 camera in its narrow mode $(10$ ". $2 \times 10$ !" 2 field of view) using natural guide star adaptive optics (Wizinowich 2013) at the Keck II telescope on four occasions: UT 2010 May 2, UT 2013 July 3, UT 2016 June 27, and UT 2017 October 10. All observations were taken in the pupil-tracking mode to employ the angular differential imaging (ADI) method (Marois et al. 2006). The star was placed behind the partly transparent 600 mas coronagraph, which has an attenuation of $7.51 \pm 0.14 \mathrm{mag}$ at $1.6 \mu \mathrm{m}$ (Bowler et al. 2015a) and enables precise image registration. The total on-source integration time of our observations was 49, 14, 35, and 30 minutes for our 2010, 2013, 2016, and 2017 epochs, respectively, and the total sky rotation of these sequences was $65^{\circ}, 13^{\circ}, 59^{\circ}$, and $48^{\circ}$. Images were corrected for cosmic rays and bad pixels, then dark subtracted and flat fielded. Details about the observations are summarized in Table 2. Further processing of the images is described in Section 4.2.

\subsection{PSF Subtraction and Astrometry}

PSF subtraction for the NIRC2 imaging data was carried out with the Locally Optimized Combination of Images algorithm (LOCI; Lafrenière et al. 2007) using the ADI processing pipeline described in Bowler et al. (2015a). Images were individually corrected for geometric distortions by bilinearly interpolating pixel values to the rectified locations based on the solution from Yelda et al. (2010) for observations taken prior to 2015 April when the Keck II AO system was re-aligned, and the solution from Service et al. (2016) was used for observations taken after pupil realignment. Each frame was then registered by fitting a 2D elliptical Gaussian to the host star located behind the partly transparent coronagraph spot. Two reductions were carried out using aggressive and conservative implementations of LOCI by varying the angular tolerance parameter used to select PSF templates $\left(N_{\delta}\right)$. Two point sources are recovered with high significance in the 2010 and 2013 epochs (Gl 758 B and bkg1; Figure 2), and three point sources are recovered in the 2016 and 2017 epochs (Gl 758 B, bkg1, and bkg2; Figure 3) in both of the reductions. We adopt the aggressive implementation for all data sets with LOCI geometric parameters of $W=5, N_{A}=300$, $g=1, N_{\delta}=0.5$, and $d r=2$ following the definitions in Lafrenière et al. (2007).

The $\mathrm{S} / \mathrm{N}$ for each point source is calculated using aperture photometry with a 5 pix aperture radius. The sky background is 
Table 2

Keck/NIRC2 Adaptive Optics Imaging of G1 758

\begin{tabular}{|c|c|c|c|c|c|c|c|c|}
\hline UT Date & $\begin{array}{l}\text { Epoch } \\
\text { (UT) }\end{array}$ & $N \times$ & $\begin{array}{l}\text { Coadds } \times t_{\exp } \\
(\mathrm{s})\end{array}$ & Filter $^{\mathrm{a}}$ & $\begin{array}{l}\text { Sep. } \\
\left({ }^{\prime \prime}\right)\end{array}$ & $\begin{array}{l}\text { P.A. } \\
\left({ }^{\circ}\right)\end{array}$ & $\begin{array}{l}\text { Detection } \\
\mathrm{S} / \mathrm{N}\end{array}$ & $\begin{array}{c}\text { PSF } \\
\text { References }\end{array}$ \\
\hline \multicolumn{9}{|c|}{ G1 758 B } \\
\hline 2013 Jul 3 & 2013.502 & 28 & $\times 30 \times 1$ & $K_{S}+\operatorname{cor} 600$ & $1.743 \pm 0.002$ & $205.7 \pm 0.2$ & 10.5 & 2 \\
\hline 2016 Jun 27 & 2016.489 & 70 & $\times 6 \times 5$ & $H+\operatorname{cor} 600$ & $1.6256 \pm 0.0019$ & $210.3 \pm 0.4$ & 20.9 & 1 \\
\hline 2017 Oct 10 & 2017.773 & 60 & $\times 6 \times 5$ & $H+\operatorname{cor} 600$ & $1.588 \pm 0.002$ & $213.5 \pm 0.3$ & 38.5 & 3 \\
\hline 2010 May 2 & 2010.333 & 98 & $\times 6 \times 5$ & $\mathrm{CH}_{4 \mathrm{~s}}+$ cor600 & $1.390 \pm 0.002$ & $222.6 \pm 0.3$ & 8.4 & 1 \\
\hline 2013 Jul 3 & 2013.502 & & $\times 30 \times 1$ & $K_{S}+\operatorname{cor} 600$ & $1.931 \pm 0.002$ & $216.1 \pm 0.2$ & 11.5 & 2 \\
\hline 2016 Jun 27 & 2016.489 & 70 & $\times 6 \times 5$ & $H+\operatorname{cor} 600$ & $2.485 \pm 0.002$ & $213.5 \pm 0.4$ & 51.1 & 1 \\
\hline 2017 Oct 10 & 2017.773 & 60 & $\times 6 \times 5$ & $H+\operatorname{cor} 600$ & $2.642 \pm 0.002$ & $212.7 \pm 0.3$ & 49.1 & 3 \\
\hline \multicolumn{9}{|c|}{ bkg2 } \\
\hline
\end{tabular}

Note. NIRC2 astrometric PSF reference stars make use of the following data sets: (1) $H$ band imaging of PYC11519+0731 from 2012 May 22 UT (Bowler et al. 2015 b); (2) $K_{S}$ band imaging of 2M22362452+4751425 from 2015 August 27 UT (Bowler et al. 2017); (3) $H$ band imaging of HD 109461 from 2017 October 10 UT.

a "cor600" refers to the 600 mas diameter focal plane coronagraph.

subtracted from the summed flux centered on the source using the mean of 100 sky measurements at the same angular radius but spanning a range of azimuthal angles surrounding (but not overlapping) the object of interest. The standard deviation of these sky values represents the background noise level, and the ratio of these two is used to determine the signal to noise of the detection. Gl $758 \mathrm{~B}$ and the two nearby background stars are detected with $\mathrm{S} /$ Ns between 10 and 51; full details can be found in Table 2.

PSF subtraction biases astrometry of point sources in processed images as a result of both over-subtraction and self-subtraction. To mitigate these effects, we follow the strategy outlined by Marois et al. (2010) of injecting a negative PSF template into the raw data and iteratively identifying the true position and flux of the sources. Three parameters were optimized using the downhill simplex AMOEBA algorithm (Nelder \& Mead 1965)—-the separation, position angle, and amplitude of the PSF template - to minimize the resulting rms in a 20 pix aperture radius at the location of the point source in the processed image. Although the host star is visible behind the coronagraph in the science frames, the mask transmission has historically been difficult to characterize in detail and may be non-uniform across the face of the occulting spot. To avoid using a potentially distorted PSF of the host star, we instead utilize unsaturated PSF templates of other stars taken in the same filter (see Table 2).

Results of the negative injection are shown in Figure 4. This procedure successfully removes most of the flux and oversubtracted azimuthal "wings," leaving only slight residual structure that likely originates from an imperfect PSF template, changing atmospheric conditions and Strehl ratios throughout the sequence (which are not taken into account in the modeling) and/or slight blurring of the PSF if substantial rotation occurs during individual exposures-something that preferentially affects sources at wider separations.

\subsection{Astrometric Error Budget}

The astrometric error budget is dominated by measurement errors in the positions of point sources; uncertainty in the residual optical distortion correction; errors in the plate scale and north orientation of the detector; and azimuthal shear caused by sky rotation within individual frames.

Our strategy for estimating point source measurement uncertainties is inspired by the method described in Rajan et al. (2017) and is intended to mimic the inverted PSF template procedure we carried out in Section 4.2. For Gl 758 B, bkg1, and bkg2, we inject a positive PSF template into the raw images at the same separation and amplitude as the objects of interest but a different position angle. We then iteratively inject an inverted PSF template of a different star into the raw data and perform local PSF subtraction with LOCI, then use AMOEBA to identify the optimal position and amplitude that minimizes the local noise. Each injection/recovery provides an estimate of the systematic difference in the separation, P.A., and amplitude of the injected (positive) object compared with what was recovered with the inverted (negative) PSF. This process is repeated ten times at equally spaced position angles for each object, and the average positional differences $\left(\sigma_{\bar{\rho}}\right.$,meas and $\sigma_{\bar{\theta}}$,meas $)$ are adopted as estimates of the positional measurement errors.

After correcting images for optical distortion effects, there remain small residual systematic positional uncertainties, $\sigma_{d}$, of about 1 mas which limit the achievable astrometric accuracy across the detector (Yelda et al. 2010; Service et al. 2016). Here we adopt one $\sigma_{d}$ term associated with the host star and one for the companion. In addition, the NIRC2 plate scale, $s$, and its associated uncertainty, $\sigma_{s}$, are taken into account and vary slightly between pre- and post-pupil realignment $\left(9.952 \pm 0.002\right.$ mas $\mathrm{pix}^{-1}$ from Yelda et al. 2010; $9.971 \pm 0.004$ mas pix $^{-1}$ from Service et al. 2016).

The final separation measurement in mas is

$$
\rho=s \bar{\rho}_{\text {meas }} \pm s \bar{\rho}_{\text {meas }}\left(\left(\frac{\sigma_{s}}{s}\right)^{2}+\left(\frac{\sigma_{\bar{\rho}, \text { tot }}}{\bar{\rho}_{\text {meas }}}\right)^{2}\right)^{1 / 2}
$$



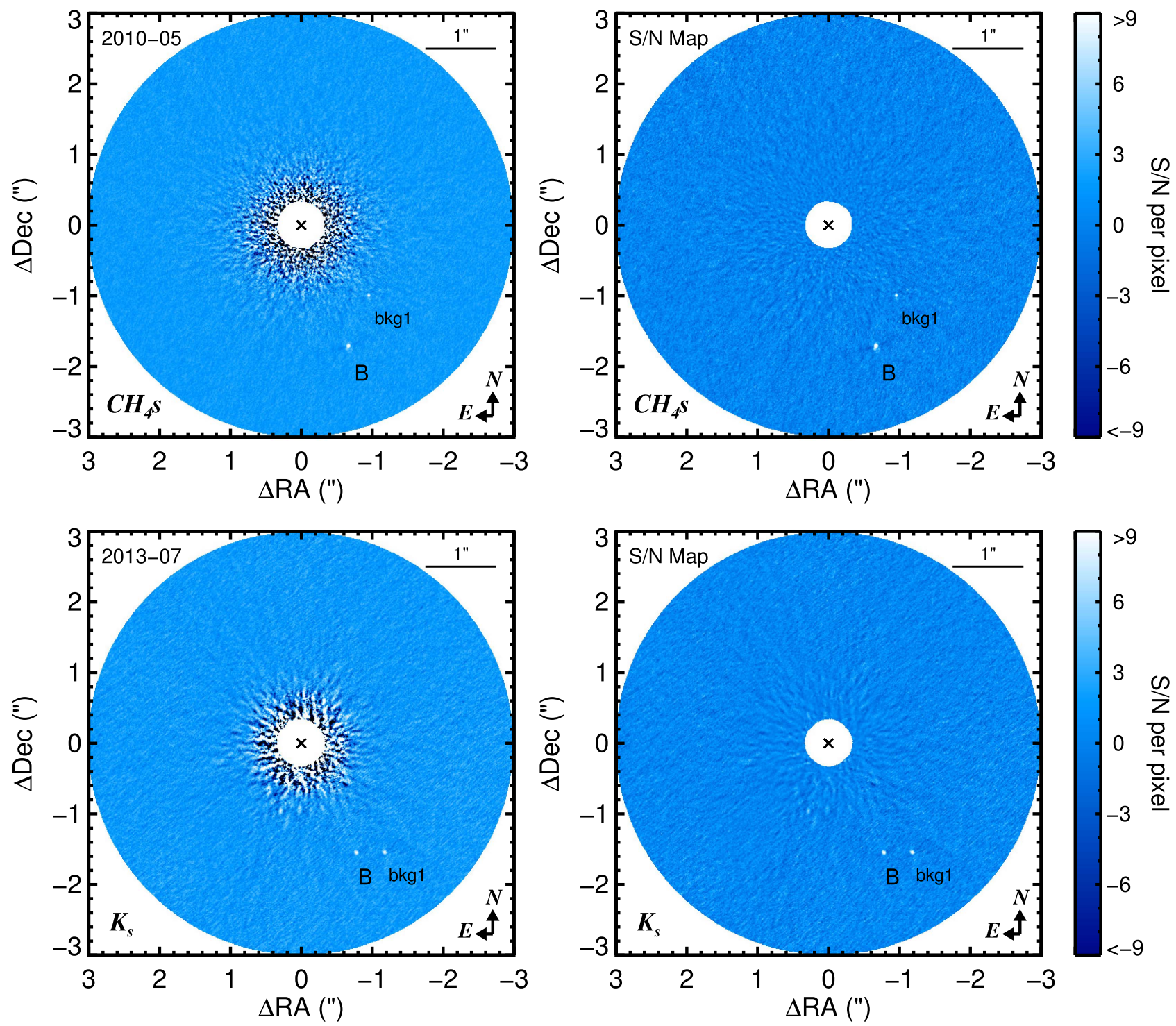

Figure 2. Final PSF-subtracted images of G1 758 taken with Keck/NIRC2 in 2010 and 2013 (upper and lower panels, respectively). Images on the left are the processed frames in units of flux $\left(\mathrm{DN} \mathrm{s}^{-1}\right)$, and images on the right are the corresponding signal-to-noise maps. The color bar on the far right corresponds to intensities in the S/N map. All images are oriented so that north is up and east is to the left. Most of the proper motion of Gl 758 is in the positive declination direction, so the background source "bkg1" moves downward over time relative to Gl 758.

where $\sigma_{\bar{\rho}, \text { tot }}$ is the combined uncertainty from our injectionrecovery exercise and from the imperfect distortion correction:

$$
\sigma_{\bar{\rho}, \text { tot }}^{2}=\sigma_{\bar{\rho}, \text { meas }}^{2}+2 \sigma_{d}^{2} .
$$

The P.A. is determined as follows:

$$
\theta=\bar{\theta}_{\text {meas }}-\theta_{\text {North }}+\theta_{\text {shear }} / 2,
$$

where $\theta_{\text {North }}$ is the rotational offset required to align the NIRC2 detector columns with north on the sky: $0.252 \pm 0.009$ for the Yelda et al. (2010) distortion solution, and $0.262 \pm 0.002$ for the Service et al. (2016) distortion solution. ${ }^{13} \theta_{\text {shear }}$ is the shear (blurring) per individual frame. To account for this, each frame

\footnotetext{
13 The position angle of celestial north with respect to the $+y$ axis for NIRC2 images taken in vertical angle (pupil tracking) mode with the narrow camera can be found using FITS header keywords: PARANG + ROTPOSN -

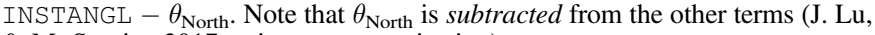
\& M. Service 2017, private communication).
}

is de-rotated to the midpoint P.A. of the exposure after PSF subtraction, and prior to coaddition of the sequence.

The error in the P.A., $\sigma_{\theta}$, includes the injection-recovery measurement uncertainty $\left(\sigma_{\theta, \text { meas }}\right)$, uncertainty in the north alignment $\left(\sigma_{\theta, \text { North }}\right)$, residual positional errors after applying the distortion solution $\left(\sigma_{\theta, d}\right)$, and the systematic error from shearing of point sources from sky rotation within each frame $\left(\sigma_{\theta, \text { shear }}\right)$ :

$$
\sigma_{\theta}=\left(\sigma_{\theta, \text { meas }}^{2}+\sigma_{\theta, \text { North }}^{2}+\sigma_{\theta, d}^{2}+\sigma_{\theta, \text { shear }}^{2}\right)^{1 / 2} .
$$

The residual positional distortion errors are about 1 mas, so here we approximate $\sigma_{\theta, d}$ as $\approx 1 \mathrm{mas} / \rho$. The dominant term in the P.A. error budget is the shear per frame, which varies among individual frames and across observation epochs. For this work we conservatively adopt half the average shear for each epoch: $0^{\circ} .30,0^{\circ} .21,0.38$, and 0.32 for our 2010,2013 , 2016, and 2017 epochs. Our final astrometry of Gl 758 B and the two background sources is listed in Table 2. Note that the 

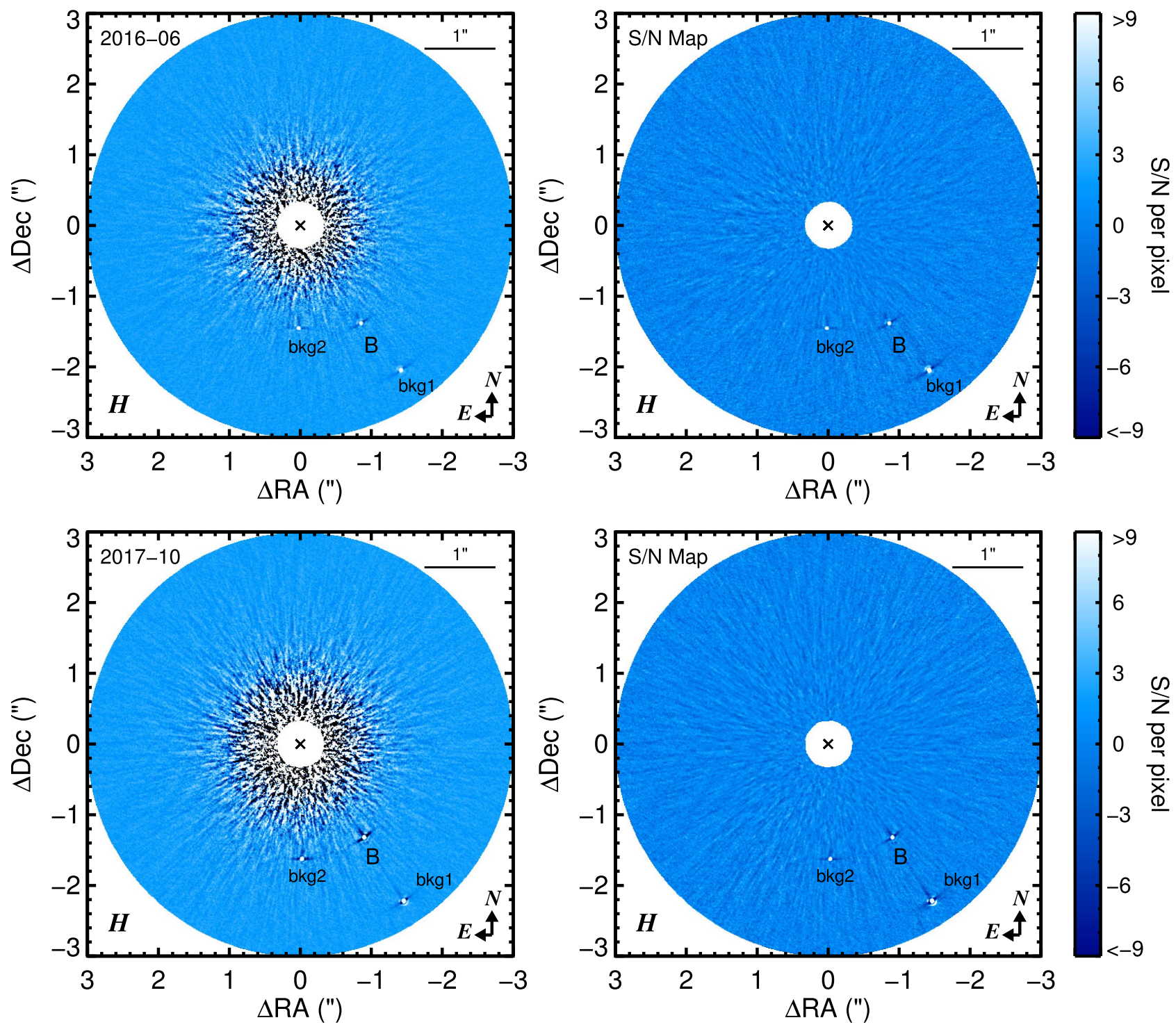

Figure 3. PSF-subtracted images of Gl 758 taken with Keck/NIRC2 in 2016 and 2017 (upper and lower panels, respectively; see Figure 2 for details). All images are oriented so that north is up and east is to the left. The background sources "bkg1" and "bkg2" are marked and move downward over time with respect to Gl 758, while the bound companion Gl 758 B orbits in a counterclockwise direction.

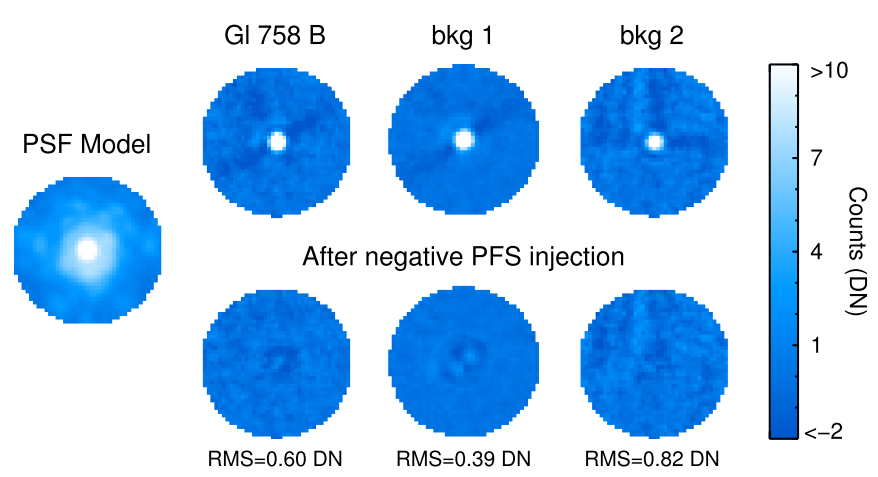

Figure 4. Example of our negative PSF injection implemented in our 2016 data set to measure unbiased astrometry of point sources. On the left is our $H$-band PSF template, in this case created using unsaturated frames of PYC J11519 +0731 taken in 2012 May from Bowler et al. (2015b). A negative version of this model is injected into raw frames, fully processed with LOCI, then iteratively adjusted in position and amplitude to minimize the noise in the final PSF-subtracted image in a 20 pix aperture. Residuals for Gl 758 B, bkg1, and bk2 are shown in the bottom three panels. resulting astrometry does not appear to be significantly sensitive to changes in the LOCI parameters used for PSF subtraction based on the same astrometric analysis with $N_{\delta}$ set to 1.5 .

\subsection{Comparison to Published Astrometry}

G1 758 B has been observed by many other telescopes and instruments over the past decade (Figure 5). The companion displays clear orbital motion; its separation has contracted from 1 ." 88 in 2009 to 1 ". 59 with our latest epoch from NIRC2 in 2017 , and has moved by $\approx 16^{\circ}$ in P.A. during that time. Our astrometry of Gl $758 \mathrm{~B}$ is broadly consistent with published values, although the separations from Nilsson et al. (2017) are significantly smaller than our measurements and those of Vigan et al. (2016) taken over the same time period. For example, our NIRC2 observations from 2013 were taken within three weeks of the 2013 July 21 data set obtained by Nilsson et al., but these two separation measurements are discrepant at the 4.3- $\sigma$ level. 

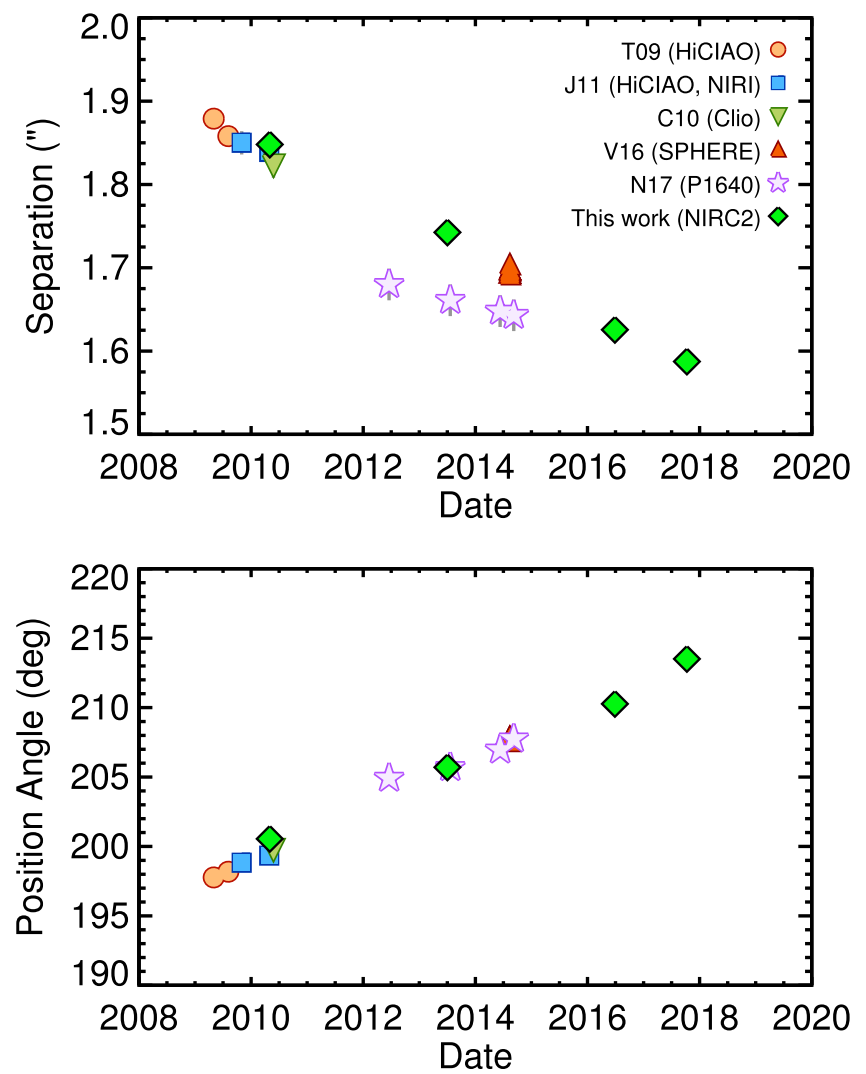

Figure 5. Relative astrometry of G1 $758 \mathrm{~B}$. The companion is approaching Gl

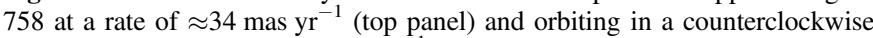
direction on the sky by $1.86 \mathrm{yr}^{-1}$ (bottom panel). Astrometry is from Thalmann et al. (2009, T09), Janson et al. (2011, J11), Currie et al. (2010, C10), Vigan et al. (2016, V16), and Nilsson et al. (2017, N17). Diamonds indicate our new epochs taken with Keck/NIRC2.

However, the P.A. measurements from Nilsson et al. are in much better agreement.

\section{Orbit and Dynamical Mass of Gl 758 B}

\subsection{Is the Acceleration Caused by Gl 758 B?}

Before carrying out a detailed joint orbit fit of the RVs and astrometry, we first demonstrate here that the observed acceleration of Gl 758 is consistent with and likely to be caused by the companion Gl $758 \mathrm{~B}$. The minimum mass of an imaged companion needed to produce an observed instantaneous acceleration $\dot{v}_{r}$ is

$$
M \approx 0.0145\left(\frac{d}{\mathrm{pc}} \frac{\rho}{\prime \prime}\right)^{2}\left|\frac{\dot{v}_{r}}{\mathrm{~m} \mathrm{~s}^{-1} \mathrm{yr}^{-1}}\right| M_{\mathrm{Jup}}
$$

where $d$ is the distance to the system in pc and $\rho$ is the projected separation in arcseconds (Torres 1999; Liu et al. 2002). Note that the generalized form of this equation includes information about the orbital elements of the system in the form of a multiplicative constant, the minimum value of which $(\approx 2.6)$ is included here in the prefactor. The measured range of accelerations and angular separations of Gl $758 \mathrm{~B}$ implies a corresponding mass range of $\approx 20-50 M_{\text {Jup. }}$. Are these reasonable values for Gl $758 \mathrm{~B}$ ? Evolutionary models from Saumon \& Marley (2008) suggest that a brown dwarf with those masses should have effective temperatures between about 430 and $1400 \mathrm{~K}$ for ages of 1-6 Gyr; this is in good agreement with the inferred effective temperatures of $600-750 \mathrm{~K}$ for $\mathrm{Gl} 758 \mathrm{~B}$ from multi-band imaging and spectroscopy (Vigan et al. 2016; Nilsson et al. 2017). Based on this consistency and the fact that the companion's orbital period must be much longer than the time baseline of the RV observations ( $\gg 20$ years), we conclude that Gl 758 B is likely the source of the acceleration.

\subsection{Joint RV and Astrometric Orbit Analysis}

We performed a joint orbit analysis of the three RV data sets and astrometry via a Markov Chain Monte Carlo (MCMC) algorithm. For this analysis we only use our NIRC2 astrometry to avoid systematic errors that may be present in previously published astrometry caused by multiple instruments and PSF subtraction strategies. We used the parallel-tempering (PT) ensemble sampler in emcee v2.1.0 (Foreman-Mackey et al. 2013) which is based on the algorithm described by Earl $\&$ Deem (2005). Thirty temperatures were adopted, of which only the coldest chain describes the posterior, together with 100 walkers to sample our 15-parameter model. Six of those parameters describe the orbit: semimajor axis $(a)$, inclination (i), P.A. of the ascending node $(\Omega)$, mean longitude $\left(\lambda_{\text {ref }}\right)$ at a reference epoch $\left(t_{\text {ref }}\right)$ of $2455197.5 \mathrm{JD}$, and finally eccentricity (e) and the argument of periastron ( $\omega$, for the host star) parameterized as $\sqrt{e} \sin \omega$ and $\sqrt{e} \cos \omega$, which avoids the Lucy-Sweeney bias toward non-zero eccentricities and imposes a uniform prior on eccentricity. We assumed a logflat prior on $a$, randomly distributed viewing angles for $i$ (i.e., a prior of $\sin i$ ), and uniform priors for the other orbit parameters. The next three parameters used in the fit were the parallax $(\pi)$ and mass of the host star $\left(M_{\text {host }}\right)$ and the mass of the companion $\left(M_{\text {comp }}\right)$. We assumed priors of $63.45 \pm 0.35$ mas on the parallax (van Leeuwen 2007), $0.97 \pm 0.02 M_{\odot}$ for $M_{\text {host }}$ (Vigan et al. 2016), and a log-flat prior for $M_{\text {comp, which is motivated }}$ by the broad range of potential masses for the companion spanning $\approx 10 M_{\text {Jup }}$ (if the system is younger than expected) to over $100 M_{\text {Jup }}$ (if the companion is an unresolved binary). The remaining six parameters are the zero points $\left(\Delta_{\mathrm{zero}, \mathrm{McD}}\right.$, $\left.\Delta_{\text {zero,Keck }}, \Delta_{\text {zero,APF }}\right)$ and jitters $\left(\sigma_{\text {jit,Keck }}, \sigma_{\text {jit,APF }}, \sigma_{\text {jit,McD }}\right)$ for the three RV data sets. The zero points are simply the offsets needed to bring the sets of relative RVs into accord with the orbit model, and the jitter terms account for small random and systematic RV epoch-to-epoch measurement errors from the star and the instrument not captured in the quoted relative RV uncertainties. We assumed uniform priors for the zero points and log-flat priors for the jitters. These are summarized in Table 3, and our complete likelihood function is as follows:

$$
\begin{aligned}
\ln (\mathcal{L})= & -0.5\left(\sum_{k=1}^{N_{\text {ast }}}\left(\frac{\rho_{k}-\rho\left(t_{k}\right)}{\sigma_{\rho, k}}\right)^{2}+\sum_{k=1}^{N_{\text {ast }}}\left(\frac{\theta_{k}-\theta\left(t_{k}\right)}{\sigma_{\theta, k}}\right)^{2}\right. \\
& \left.+\sum_{j=1}^{N_{\text {inst }}} \sum_{k=1}^{N_{\mathrm{RV}}} \frac{\left(\mathrm{RV}_{\mathrm{rel}, k}+\Delta_{\mathrm{zero}, j}-\mathrm{RV}\left(t_{k}\right)\right)^{2}}{\sigma_{\mathrm{RV}, k}^{2}+\sigma_{\mathrm{jit}, j}^{2}}\right) \\
& +\ln (\sin (i))-\ln (a)-\ln \left(M_{\mathrm{sec}}\right) \\
& -0.5\left(\frac{\pi-63.45 \mathrm{mas}}{0.35 \text { mas }}\right)^{2} .
\end{aligned}
$$

We are able to fit for the RV jitter because of the numerous independent data points that sample its orbit in each data set. In other words, there are many degrees of freedom in the RV 
Table 3

MCMC Posteriors for the Orbit of G1 758B

\begin{tabular}{|c|c|c|c|c|}
\hline Property & Mode $\pm 1 \sigma$ & Best fit & $95.4 \%$ c.i. & Prior \\
\hline \multicolumn{5}{|c|}{ Fitted parameters } \\
\hline Companion mass $M_{\text {comp }}\left(M_{\mathrm{Jup}}\right)$ & $42_{-7}^{+19}$ & 55 & 33,106 & $1 / M$ (log-flat) \\
\hline Host-star mass $M_{\text {host }}\left(M_{\odot}\right)$ & $0.967_{-0.018}^{+0.022}$ & 0.969 & $0.929,1.009$ & $0.970 \pm 0.020 M_{\odot}($ Gaussian $)$ \\
\hline Parallax (mas) & $63.39_{-0.32}^{+0.37}$ & 63.51 & $62.73,64.13$ & $63.45 \pm 0.35$ mas (Gaussian) \\
\hline Semimajor axis $\alpha$ (mas) & $1340_{-80}^{+170}$ & 1350 & 1210,1820 & $1 / \alpha(\log -$ flat $)$ \\
\hline Inclination $i\left({ }^{\circ}\right)$ & $28_{-10}^{+12}$ & 27 & 10,49 & $\sin (i), 0^{\circ}<i<180^{\circ}$ \\
\hline$\sqrt{e} \sin \omega$ & $-0.05_{-0.09}^{+0.11}$ & -0.06 & $-0.26,0.24$ & uniform \\
\hline$\sqrt{e} \cos \omega$ & $-0.76_{-0.03}^{+0.08}$ & -0.75 & $-0.82,-0.47$ & uniform \\
\hline Mean longitude at $t_{\mathrm{ref}}=2455197.5 \mathrm{JD}, \lambda_{\text {ref }}\left({ }^{\circ}\right)$ & $72 \pm 8$ & 72 & 52,89 & uniform \\
\hline PA of the ascending node $\Omega\left({ }^{\circ}\right)$ & $175 \pm 5$ & 174 & 163,183 & uniform \\
\hline McDonald RV zero point $\left(\mathrm{m} \mathrm{s}^{-1}\right)$ & $61 \pm 19$ & 59 & 27,108 & uniform \\
\hline Keck RV zero point $\left(\mathrm{m} \mathrm{s}^{-1}\right)$ & $50_{-19}^{+20}$ & 50 & 17,98 & uniform \\
\hline APF RV zero point $\left(\mathrm{m} \mathrm{s}^{-1}\right)$ & $44_{-21}^{+18}$ & 42 & 9,90 & uniform \\
\hline McDonald RV jitter $\sigma_{\mathrm{McD}}\left(\mathrm{m} \mathrm{s}^{-1}\right)$ & $3.1_{-0.6}^{+0.8}$ & 3.0 & $1.6,4.6$ & $1 / \sigma$ (log-flat) \\
\hline Keck RV jitter $\sigma_{\text {Keck }}\left(\mathrm{m} \mathrm{s}^{-1}\right)$ & $2.33_{-0.18}^{+0.17}$ & 2.32 & $2.00,2.70$ & $1 / \sigma$ (log-flat) \\
\hline APF RV jitter $\sigma_{\mathrm{APF}}\left(\mathrm{m} \mathrm{s}^{-1}\right)$ & $2.46_{-0.17}^{+0.18}$ & 2.44 & $2.13,2.84$ & $1 / \sigma$ (log-flat) \\
\hline \multicolumn{5}{|c|}{ Computed properties } \\
\hline Orbital period $P(\mathrm{yr})$ & $96_{-9}^{+21}$ & 97 & 79,153 & $\cdots$ \\
\hline Semimajor axis $a(\mathrm{au})$ & $21.1_{-1.3}^{+2.7}$ & 21.3 & $18.9,28.7$ & $\cdots$ \\
\hline Eccentricity $e$ & $0.58_{-0.11}^{+0.07}$ & 0.57 & $0.26,0.67$ & $\cdots$ \\
\hline Argument of periastron $\omega\left(^{\circ}\right)$ & $184_{-9}^{+8}$ & 184 & 153,201 & $\cdots$ \\
\hline Time of periastron $T_{0}=t_{\mathrm{ref}}-P \frac{\lambda-\omega}{360^{\circ}}$ (JD) & $2465800_{-800}^{+2000}$ & 2466300 & 2464800,2470500 & $\cdots$ \\
\hline Mass ratio $q=M_{\mathrm{comp}} / M_{\mathrm{host}}$ & $0.042_{-0.008}^{+0.018}$ & 0.054 & $0.032,0.105$ & $\cdots$ \\
\hline
\end{tabular}

model of the host star. In contrast, with only four epochs of companion astrometry we do not have the same ability to fit for additional astrometric errors. Therefore, we performed an initial orbit fit using the nominal astrometric errors and examined the residuals. The rms of the separation measurements was 4.3 mas about this initial best-fit orbit and $\chi^{2}=14.6$, while for the P.A.s the rms was 0.34 with $\chi^{2}=3.0$. As a point comparison, when we simply fit a line to separation and P.A. as a function of time, we found similar rms values of of 4.7 mas $\left(\chi^{2}=17.5\right)$ and $0.45\left(\chi^{2}=4.9\right)$. Given that the RVs constrain some of the same orbit parameters that are relevant to the astrometric fit, it is not obvious what number of degrees of freedom is correct to assume here. If we assume two degrees of freedom in each, then $p\left(\chi^{2}\right)=0.0007$ for the separations and $p\left(\chi^{2}\right)=0.22$ for the P.A.s. Ultimately we add 4.3 mas in quadrature to our separation measurements, resulting in effective errors of $\approx 5$ mas at every epoch. We do not add any additional error to our P.A. uncertainties as these are already substantially larger $\left(\approx 0^{\circ} .3\right.$, or $\approx 10$ mas $)$ and the $\chi^{2}$ value is not unreasonable. The source of the 4-5 mas epoch-to-epoch uncertainties in our separation measurements is not known, so it may represent a fundamental floor to astrometry derived from ADI sequences with the NIRC2 coronagraph.

The initial state of the PT-MCMC sampler was determined using a Monte Carlo rejection sampling analysis similar to the method used in Dupuy et al. (2016). First, $2 \times 10^{6}$ randomly distributed orbital periods $\left(10^{4} d<P<10^{7} d\right)$, eccentricities, and times of periastron passage $\left(T_{0}\right)$ were drawn. Using the formalism of Lucy (2014), we computed the corresponding set of $a, i, \omega$, and $\Omega$ that best fit the astrometry for each of these trials. For each trial, we computed the $\chi_{\text {ast }}^{2}$ of the trial orbit's predicted astrometry and our measured astrometry. To incorporate the RVs, we assumed at this stage that each data set could be represented as a simple linear trend with time. For each orbit trial, we computed the instantaneous slope of the host-star RV at the mean epoch for each RV data set. (In our actual PT-MCMC runs, we fit all the individual relative RV measurements directly.) Because each trial has $a$ and $P$ independent of assumptions about mass, each trial also effectively samples an associated total mass through $M_{\text {tot }} \propto$ $a^{3} / P^{2}$. We computed the mass ratio that would best bring each orbit's RV slopes into agreement with the measured slopes and then computed the RV zero points needed to bring the measured relative $\mathrm{RVs}$ into agreement. Because there are multiple RV slopes to reproduce, the agreement is not perfect for a given orbit trial, and we computed the $\chi_{\mathrm{RV}}^{2}$ of the trial orbit's predicted RV slopes and our measured RV slopes. Finally, we computed the $\chi_{\text {mass }}^{2}$ of the trial orbit's predicted host-star mass with the estimated mass of $0.97 M_{\odot}$ from Vigan et al. (2016), with an inflated uncertainty of $\pm 0.20 M_{\odot}$ to allow us to perform orbit fits with no mass prior as well. We combined these constraints into $\chi_{\text {tot }}^{2}=\chi_{\mathrm{ast}}^{2}+\chi_{\mathrm{RV}}^{2}+$ $\chi_{\text {mass }}^{2}$, computed rejection probabilities of $p_{\text {rej }}=1-\exp$ $\left(-\left(\chi_{\text {tot }}^{2}-\min \left(\chi_{\text {tot }}^{2}\right)\right) / 2\right)$, and then drew random samples to pass on based on $p_{\text {rej }}>\mathcal{U}(0,1)$, where $\mathcal{U}(0,1)$ was a uniformly distributed, randomly drawn number ranging from 0 to 1 .

In our PT-MCMC analysis, we experimented with different chain lengths and found that after $\sim 10^{5}$ steps our 100-walker chains had clearly stabilized in the mean and rms of the posterior for each of the parameters. We saved every 20th step of our chains and discarded the first $50 \%$ of the chain as the burn-in portion, leaving $2.5 \times 10^{5} \mathrm{PT}-\mathrm{MCMC}$ samples in the cold chain. Table 3 lists information on the posterior distributions of our fitted parameters, as well as parameters that are directly computed from them. To compute the modes 
of our distributions we binned the posterior and found the bin with the most elements. The $1-\sigma$ and 2- $\sigma$ confidence intervals are computed as the minimum range in that parameter that contains $68.3 \%$ and $95.4 \%$ of the values, respectively. The quoted best-fit solution is the one with the maximum likelihood, which includes the prior.

Figure 6 displays the companion mass posterior and the most relevant parameter correlations, and Figure 7 shows several of the other marginalized posterior distributions from our fit. As expected, the inclination is highly correlated with companion mass (i.e., $M_{\text {comp }} \sin (i)$ is well constrained from the RV orbit). The companion mass posterior extends to high masses $\left(>80 M_{\text {Jup }}\right.$ ) that are likely unphysical assuming that Gl $758 \mathrm{~B}$ is a single object. This high-mass tail corresponds to low inclinations $\left(i \lesssim 20^{\circ}\right)$ that our astrometry cannot rule out, high eccentricities $(e \gtrsim 0.6)$, and short periods $(P \lesssim 100 \mathrm{yr})$. The companion mass posterior has a sharp lower limit of $30.5 M_{\text {Jup }}$ at the 4- $\sigma$ level. ${ }^{14}$ Figures 8 and 9 show our orbit solutions relative to our RV and astrometric data. The sky-projected and de-projected solution for Gl 758 is displayed in Figure 10, where orbits are drawn from the MCMC posteriors and are color-coded according to the corresponding companion mass from low mass (pink) to high mass (green).

\section{Results and Discussion}

\subsection{Nature of bkg2}

Vigan et al. (2016) identified a new point source near Gl 758 at a separation of $\approx 1$ !" 1 based on observations taken with SPHERE in 2014. They found that the photometry of this new object is broadly consistent with the colors of $\mathrm{L}$ dwarfs, raising the possibility that this could be a second companion in this system. This object is easily recovered in our 2016 and 2017 data sets at wider separations of 1."46 and 1."62. Together with the single-epoch detection from Vigan et al. taken in 2014, this object closely follows the expected motion for a background star (Figure 11) and appears to be unassociated with Gl 758.

\subsection{Bolometric Luminosity of $G l 758$ B}

Despite extensive efforts to characterize this system with follow-up photometry and spectroscopy, only a single bolometric luminosity estimate by Currie et al. (2010) exists in the literature: $\log \left(L / L_{\odot}\right)=-6.1_{-0.2}^{+0.3}$ dex. To improve on this value we use use existing near-infrared measurements to constrain the 1-3 $\mu \mathrm{m}$ spectral energy distribution together with atmospheric models for a bolometric correction. We first anchor the $1.0-1.75 \mu \mathrm{m}$ spectrum of Gl 758 B from Nilsson et al. (2017) by flux calibrating the P1640 observations to the $H$-band apparent magnitude from Thalmann et al. (2009). To this we add the photometry from Vigan et al. (2016) and Janson et al. (2011) to directly account for the comparably high $K$-band flux from this object. A solar-metallicity BT-Settl "CIFIST2011" atmospheric model with $T_{\text {eff }}=650 \mathrm{~K}$ and $\log g=5.0 \mathrm{dex}$ is used for the long-wavelength bolometric correction $(2.5-500 \mu \mathrm{m})$ by flux-calibrating the model to the $L^{\prime}$-band photometry from Janson et al. (2011). This approach also agrees with the $M_{S}$-band upper limit from Janson et al.

\footnotetext{
14 Note that this lower limit on the companion mass is relatively insensitive to changes in priors. We also ran our joint fit after removing the prior on the host star mass; the resulting mode of the companion mass distribution is $43 M_{\text {Jup }}$ with a $95 \%$ credible interval of $33-131 M_{\text {Jup }}$ and a lower limit of $31.7 M_{\text {Jup }}$ (at the 4- $\sigma$ level).
}

(2011). The same model is used for the short-wavelength correction $(0.1-1.0 \mu \mathrm{m})$ by scaling that region to the blue end of the P1640 spectrum. Uncertainties in the spectral measurements, photometry, and flux calibration scale factors for the model and spectrum are all accounted for in a Monte Carlo fashion by integrating under new realizations of the complete $0.1-500 \mu \mathrm{m}$ spectrum. This procedure yields a bolometric luminosity of $\log \left(L / L_{\odot}\right)=-6.07 \pm 0.03$ dex for Gl $758 \mathrm{~B}$. To assess possible systematic errors, we experimented with alternative atmospheric models from the same grid with effective temperatures of $600 \mathrm{~K}$ and $700 \mathrm{~K}$. The results following the same procedure are within 0.02 dex of the value we obtained with the $650 \mathrm{~K}$ model, which is smaller than the impact of random measurement errors.

\subsection{Comparison with Evolutionary Models}

With a measured luminosity, age, and dynamical mass, Gl $758 \mathrm{~B}$ offers a rare opportunity to test substellar evolutionary models. For this analysis we begin with the assumption that the age range spans 1-6 Gyr following Vigan et al. (2016), but ultimately re-evaluate this constraint based on recent results from isochrone fitting. We select a variety of publicly available models from the literature for this exercise: the Cond models from Baraffe et al. (2003); three versions of evolutionary models from Saumon \& Marley (2008) with no clouds ("SMNC"), a hybrid prescription for the evolution of clouds at the $\mathrm{L} / \mathrm{T}$ transition ("SM-Hybrid"), and the retention of thick clouds at all temperatures ("SM-f2"); and the grid from Burrows et al. (1997). All have solar compositions. These models mainly differ in their treatment of atmospheric clouds and molecular opacities, which act as boundary conditions that control the evolution of brown dwarfs and giant planets as these objects radiatively cool over time (see, e.g., Burrows et al. 2001 and Marley \& Robinson 2015 for detailed reviews).

Our approach for comparing the models to the observations utilizes a one-tailed hypothesis test. We adopt a null hypothesis in which the posterior probability density function for the dynamical mass of Gl 758 B is statistically consistent with the inferred mass distribution from evolutionary models at some threshold probability; we choose $0.3 \%$ (within $3-\sigma$ ) for this study. In other words, we calculate the probability that random draws from the dynamical mass distribution differ from the inferred model-based mass distribution. If these two values disagree by at least 0.997 , then the null hypothesis is rejected and the two distributions are considered to be inconsistent with each other.

For each set of evolutionary models, we randomly draw ages from a uniform distribution $(\tau=\mathcal{U}(1,6) \mathrm{Gyr})$ and luminosities from a log-normal distribution $\left(\log \left(L / L_{\odot}\right)=\mathcal{N}(\mu=-6.07\right.$, $\sigma=0.03$ ) dex); a visual reference is shown in Figure 12 for the Burrows models. For each $\{\tau, L\}$ pair we find the corresponding mass by finely interpolating the model grid. This Monte Carlo process is repeated of order $10^{6}$ times to create a distribution of expected masses for each model. The predicted and dynamical mass distributions are then quantitatively compared for consistency.

Results from this analysis are listed in Table 4 and illustrated in Figure 13. Based on the input age and luminosity distributions together with our threshold criterion for agreement, only the Burrows models are formally consistent with the dynamical mass distribution, which peaks at $42 M_{\text {Jup }}$ and has a robust (4- $\sigma$ ) lower limit of $30.5 M_{\text {Jup. However, even the }}$ formal agreement with the Burrows models is marginal and a 

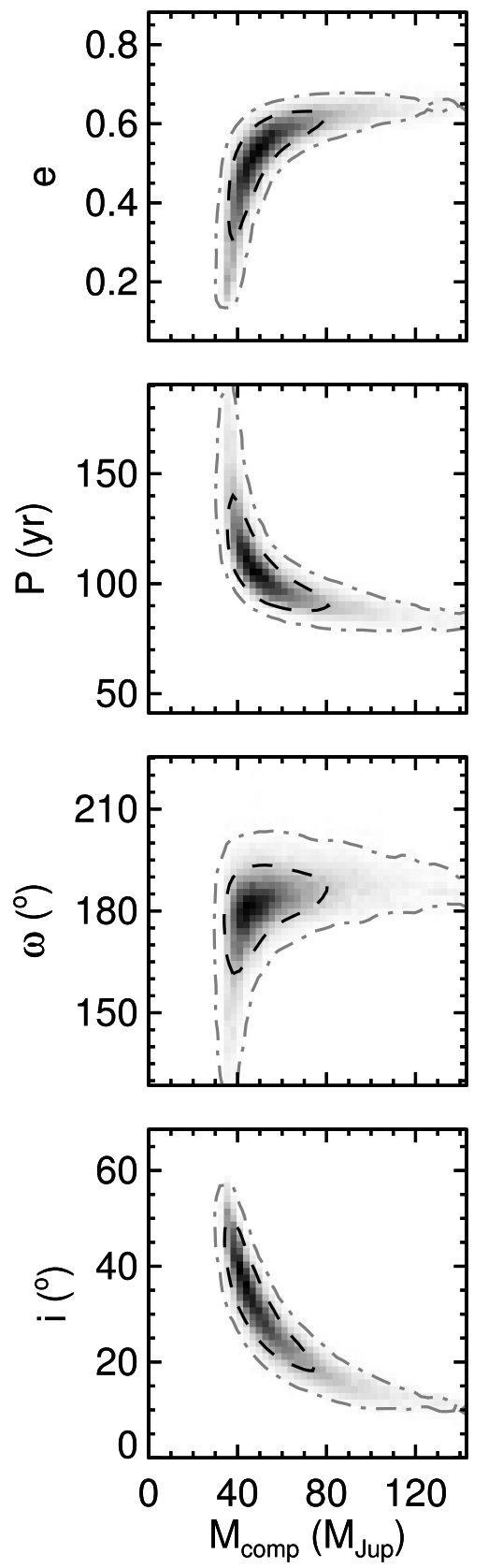
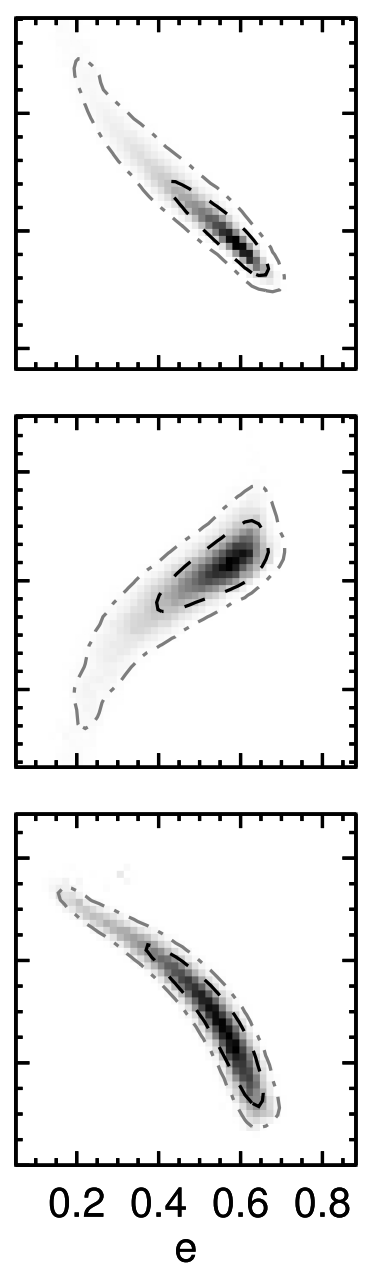

Gl 758B
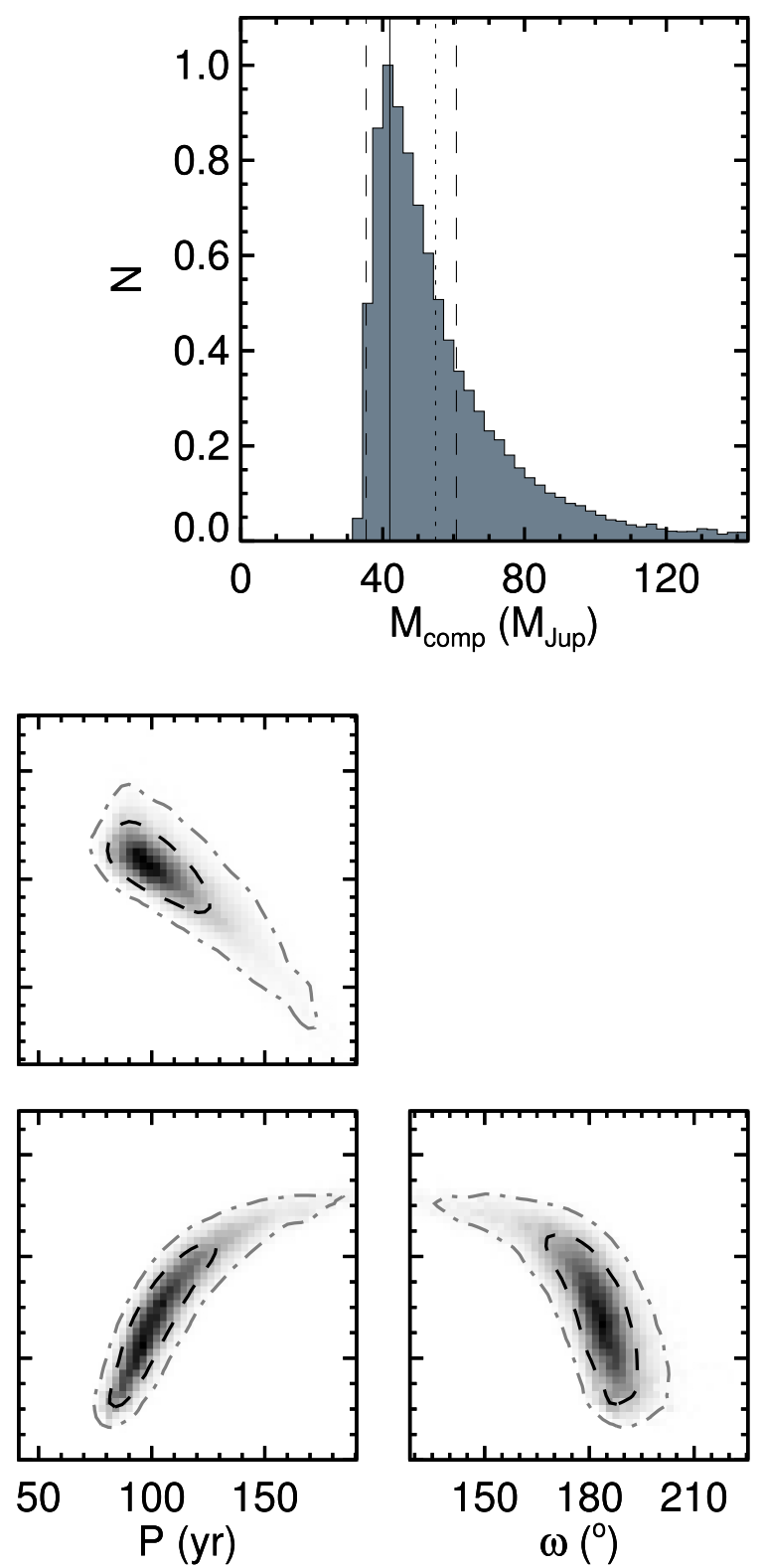

Figure 6. Posterior distributions of orbital parameters from our MCMC analysis. Grayscale images show the relation between the companion mass $\left(M_{\text {comp }}\right)$ and the several of the most correlated parameters: inclination, eccentricity, period, and the argument of periastron. Contours indicate the regions containing 68.3\% (black dashed lines) and $95.4 \%$ (gray dashed-dotted lines). The histogram (top right) shows the marginalized posterior of $M_{\text {comp }}$ with the mode (solid line), best-fit (dotted line), and $68.3 \%(1 \sigma)$ interval (dashed lines) indicated.

slightly lower threshold for consistency would have rejected the null hypothesis; random draws from the dynamical mass distribution result in higher masses $99 \%$ of the time. All other models fail our hypothesis test. The SM-f2 models disagree the most with the dynamical mass of G1 758 B. By retaining clouds to temperatures well below the rainout limit for various grains, this prescription is the most unrealistic for T dwarfs like Gl 758 B (which is expected to be cloud-free) so this result is unsurprising.

The general tension between the models and our dynamical mass measurement may point to physics not yet incorporated into current substellar models, which could originate from several sources: interior physics and thermal structure, sources of atmospheric opacity and their evolution with temperature, initial entropy and accretion history of Gl 758 B, or ill-matched metallicities of the models and the companion. On the other hand, it is also possible that the discrepancy originates from the observational side, most likely with the age of the system. For our default analysis we adopted the 1-6 Gyr estimate by Vigan et al. (2016) based on isochrone fitting of the host star (which resulted in younger ages of $\approx 1-4 \mathrm{Gyr}$ ) and activity indicators (which resulted in older ages of $\approx 3-8 \mathrm{Gyr}$ ). Older ages result in higher predicted masses for the same luminosity, so this could also be a natural explanation for the disagreement.

To explore the possibility of an older age or a systematic offset in the luminosities of the models, we perform a series of tests to identify the minimum compatible ages and the minimum compatible luminosities that render the inferred and 

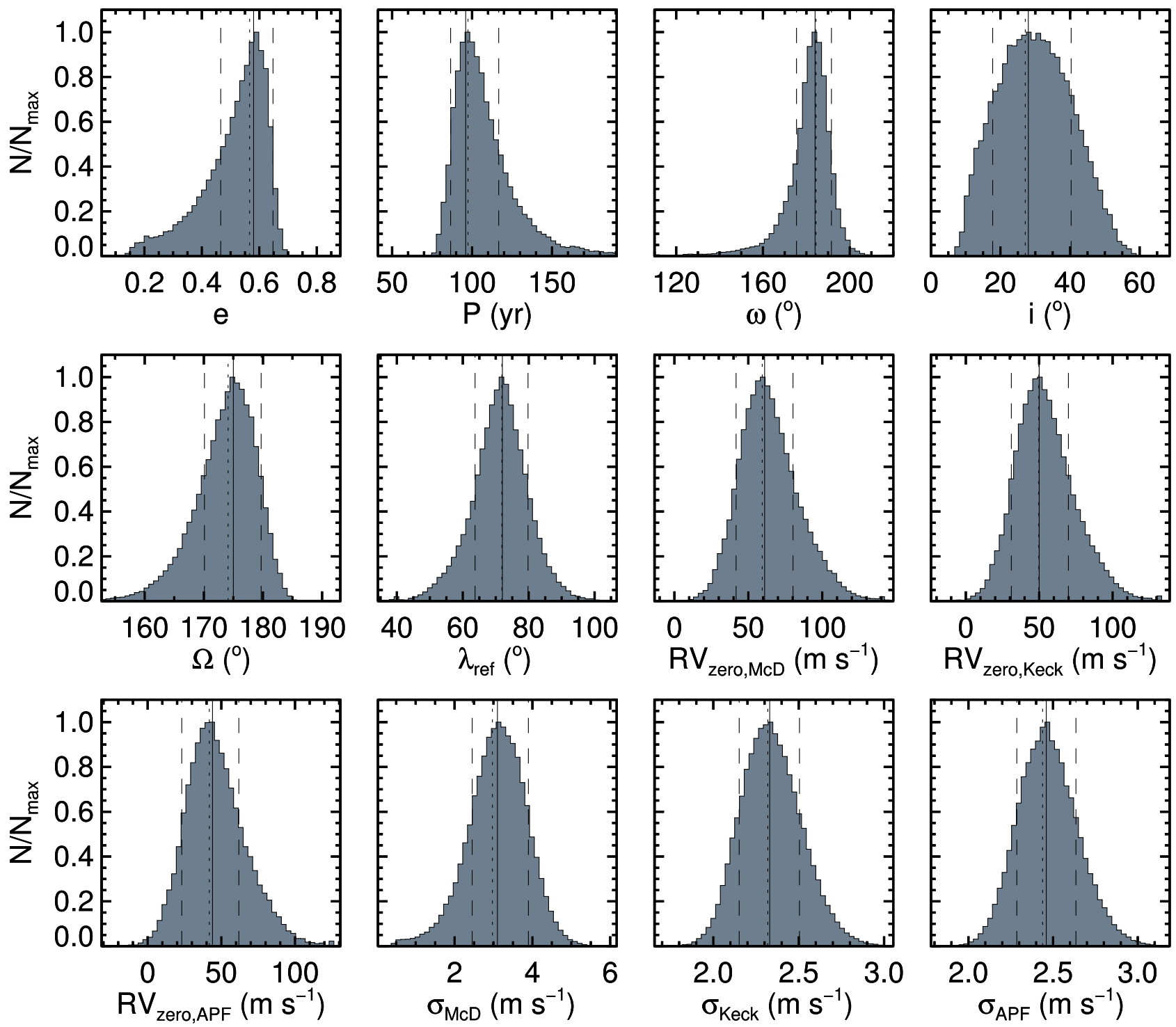

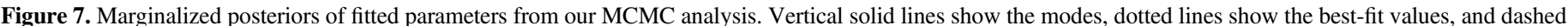
lines show the $68.3 \%(1 \sigma)$ intervals.

dynamical mass distributions into agreement. For the former, we begin by randomly drawing masses from interpolated evolutionary model grids following a normal distribution in log-luminosity $(\mathcal{N}(-6.07,0.03)$ dex $)$ and fixing the starting age at $1.0 \mathrm{Gyr}$. Using the same threshold requirement of $0.3 \%$, we compare the inferred and dynamical mass distributions for consistency. This process is then repeated by increasing the age in $0.1 \mathrm{Gyr}$ steps until the mass distributions agree at the threshold level. The results are summarized in Table 4; the Cond, SM-NC, and SM-Hybrid models all imply similar ages of $\gtrsim 6$ Gyr. The SM-f2 grid is only consistent with the dynamical mass for extremely old ages of $\gtrsim 9 \mathrm{Gyr}$, and the Burrows models agree for ages beyond $4.4 \mathrm{Gyr}$.

A similar process is carried out to identify the minimum luminosity consistent with the measured mass. We randomly draw masses based on a uniform distribution of ages $(\mathcal{U}(1,6)$ Gyr) and a fixed starting luminosity of $-6.50 \mathrm{dex}$, then test the inferred and dynamical mass distributions for consistency. The luminosity is increased in increments of $0.01 \mathrm{dex}$ until agreement is reached. If the 1-6 Gyr age estimate is correct, that would imply that the Cond, SM-NC, SM-Hybrid, and
SM-f2 evolutionary models are overluminous by 0.11 dex, $0.12 \mathrm{dex}, 0.15 \mathrm{dex}$, and $0.31 \mathrm{dex}$, respectively (Table 4). This potential discrepancy is in the opposite sense from results by Dupuy et al. (2009, 2014), who found that substellar cooling models under-predict the luminosities of brown dwarfs with dynamical masses by $\approx 0.2-0.4 \mathrm{dex}$, at least at relatively young age of $\approx 0.5-1$ Gyr.

Altogether, the most likely culprit for the disagreement in mass probably resides in the age of Gl 758. Older ages of 6-9 Gyr would readily put the predicted and dynamical distributions in excellent agreement and are indeed suggested from the low activity level, lack of X-ray emission, and slow projected rotational velocity of Gl 758 (Mamajek \& Hillenbrand 2008; Thalmann et al. 2009; Vigan et al. 2016). Although there are a wide range of age estimates for the host star from isochrone fitting in the literature, more recent analyses are converging on an older value that agrees better with activity indicators. For example, a recent study by Luck (2017) found an average age of 7.5 Gyr (with a range of 5.3 Gyr about that value) using four sets of isochrones, and Brewer et al. (2016) found an isochronal age 

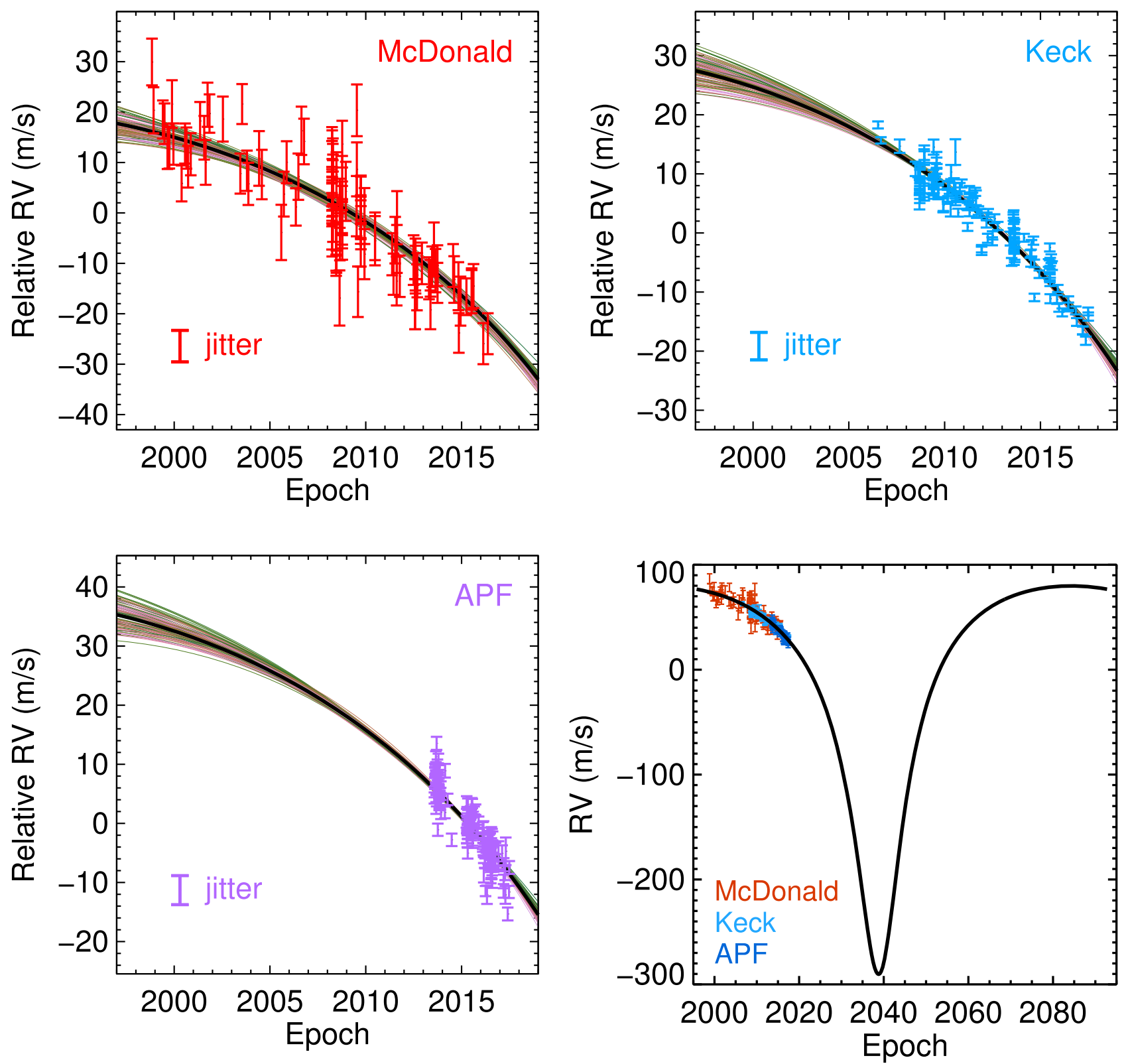

Figure 8. Relative RV measurements of Gl 758 from McDonald (top left), Keck (top right), and APF (bottom left). Randomly drawn orbit solutions from our MCMC posterior are displayed as thin colored lines, coded by the companion mass from low (pink) to high (green). Each RV data set has its own RV zero point associated with each orbit solution, allowing the absolute, barycentric RVs predicted from the orbit to be plotted as relative RVs here. The best-fit orbit solution is shown as a thick black line, and the error bar in the lower left of each plot shows the best-fit jitter (i.e., the additional RV error that is added in quadrature to the displayed measurements during our MCMC analysis). Each RV data set is shown with its best-fit zero point added to bring the relative RVs into the barycentric frame. Jitter has not been added to the plotted error bars. The RV measurements jointly show a nonlinear trend, indicating that the acceleration of the host star is changing with time. The bottom-right panel shows the RVs relative to the best-fit orbit spanning a complete orbital cycle ( $97 \mathrm{yr})$.

of $7.5 \mathrm{Gyr}$ with a range of $4.6-10.4 \mathrm{Gyr}$ using the Yonsei-Yale models.

\subsection{Limits on Planetary Companions}

We searched the residual RVs for closer-in planet candidates using a Lomb-Scargle periodogram after removing the best-fit orbital solution of Gl 758 B. The strongest power from $1-10^{4}$ days is at a period of 245.5 days, but that potential signal has a false alarm probability of $0.4 \%$ and its corresponding velocity semi-amplitude is at the level of the noise in the data, so it is unlikely to be real. No frequencies have powers that exceed our threshold false alarm probability of $0.1 \%$ for planet detection. We conclude that there is no convincing evidence of any close-in planet candidates in our data.

Detection limits are quantified using injection-recovery tests as described in Howard \& Fulton (2016). Synthetic planets on circular orbits are sequentially injected into the RV residuals by randomly drawing pairs of minimum mass and period surrounding the detection threshold. A periodogram is used to search for planets within each artificial data set with a $1 \%$ false alarm probability threshold for recovery and the requirement of a similar period and phase as the injected planet. Results are shown in Figure 14. Gl 758 is devoid of 

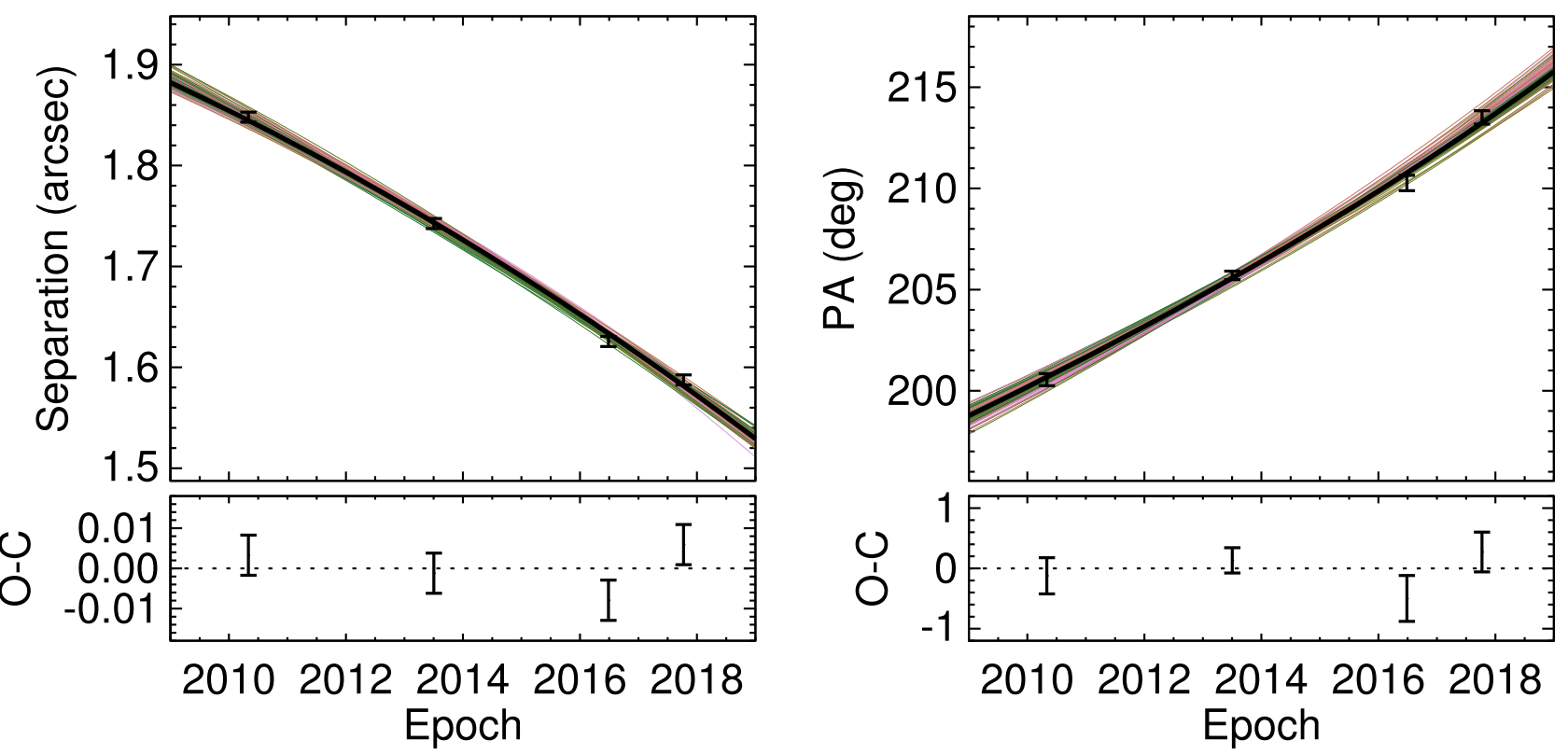

Figure 9. Separation (left) and P.A. (right) measurements of Gl 758 B relative to the primary. Randomly drawn orbit solutions from our MCMC posterior are displayed as thin colored lines, coded by the companion mass from low (pink) to high (green). The best-fit orbit solution is shown as a thick black line.
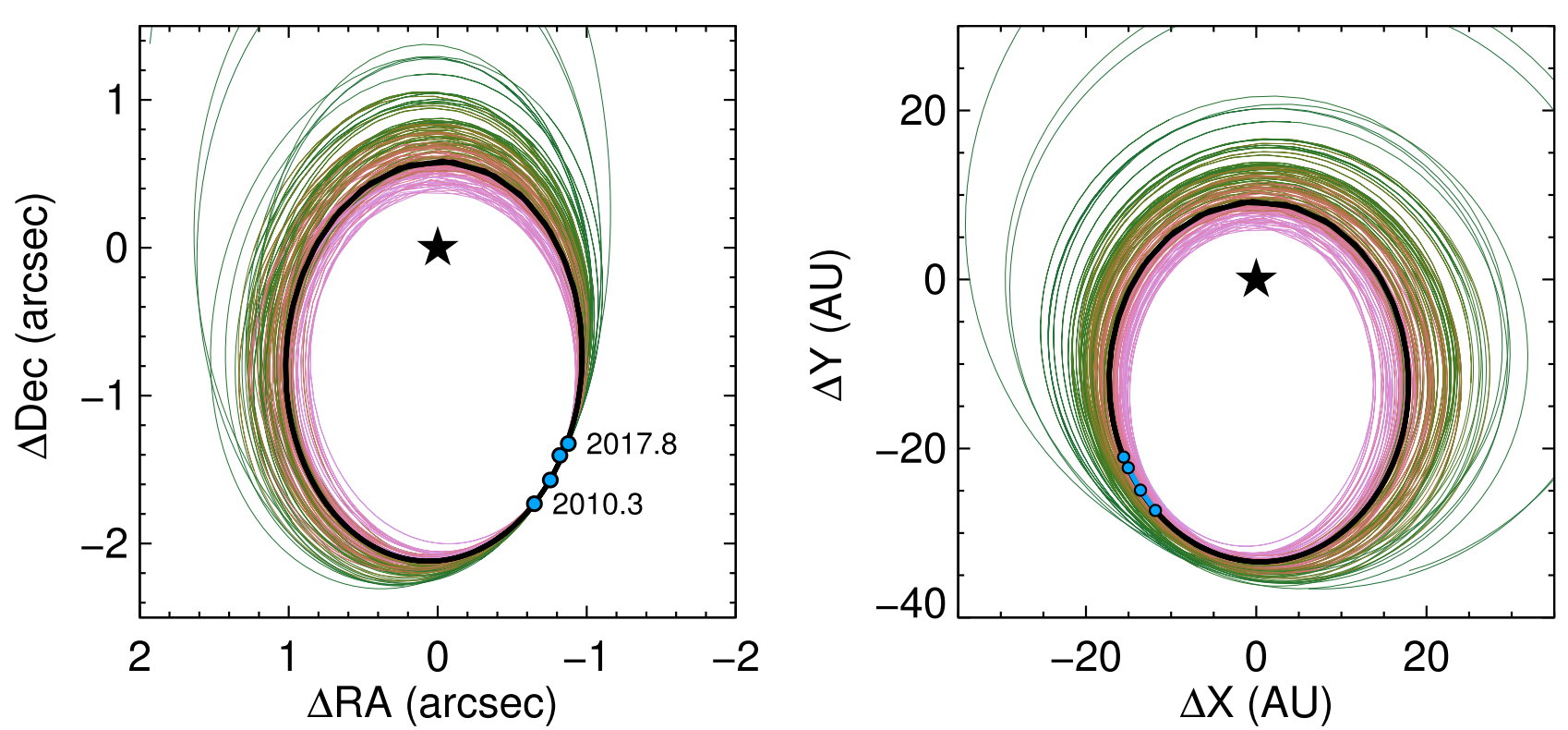

Figure 10. Left: astrometry of Gl 758 B (blue circles) relative to the primary (black star) shown alongside randomly drawn orbit solutions from our MCMC posterior. Right: the same orbit solutions viewed face-on (i.e., $i$ set to zero) with the epochs of our astrometry marked in blue. The best-fit orbit solution is shown as a thick black line. The randomly drawn orbits are color coded according to the companion mass, from low mass (pink) to high mass (green). Higher companion mass is strongly correlated with smaller, more eccentric, and closer to face-on orbits.

close-in giant planets $\left(\gtrsim 100 M_{\oplus}\right)$ within $10 \mathrm{au}$, sub-Saturns $\left(\approx 10-100 M_{\oplus}\right)$ within 3 au, and super-Earths $\left(\approx 2-10 M_{\oplus}\right)$ interior to 0.1 au.

Given its periastron passage of $8.9_{-2.0}^{+3.7}$ au, it is likely that $\mathrm{Gl}$ $758 \mathrm{~B}$ has impacted the formation efficiency and dynamical stability of closer-in planets in this system. If Gl 758 B formed relatively quickly ( $\lesssim 1 \mathrm{Myr}$ ), perhaps from turbulent fragmentation (e.g., Bate 2009) with subsequent migration to its current orbit, this implies that the circumprimary protoplanetary disk would have been truncated between $\sim 0.2$ and $0.35 a$, or about 4-7 au (Artymowicz \& Lubow 1994). The lack of planets outside of this region is not unexpected, but planet formation interior to this region may still have been possible (e.g., Kepler444; Campante et al. 2015; Dupuy et al. 2016).

However, the lack of planets at small orbital distances from G1 758 is not particularly unusual compared to the statistical properties of planets orbiting GK dwarfs in general. For example, Cumming et al. (2008) find that about $10 \%$ of Sunlike stars host giant planets with minimum masses between 0.3 and $10 M_{\text {Jup }}$ within $\approx 3$ au. This value increases to about $14 \%$ by extrapolating the planet period distribution out to $10 \mathrm{au}$. Wittenmyer et al. (2016) infer the frequency of Jupiter analogs between 3 and 7 au around solar-type stars to be $6.2_{-1.6}^{+2.8} \%$ (see also Wittenmyer et al. 2011; Zechmeister et al. 2013). Petigura 
Table 4

Predictions from Evolutionary Models

\begin{tabular}{|c|c|c|c|c|c|c|c|}
\hline \multicolumn{3}{|c|}{ Constraint } & \multicolumn{5}{|c|}{ Model } \\
\hline $\begin{array}{l}\text { Age } \\
\text { (Gyr) }\end{array}$ & $\begin{array}{l}\text { Luminosity } \\
\left(\log \left(L / L_{\odot}\right)\right)\end{array}$ & $\begin{array}{l}\text { Mass } \\
\left(M_{\text {Jup }}\right)\end{array}$ & Cond & SM-NC & SM-Hybrid & SM-f2 & Burrows \\
\hline & & & \multicolumn{5}{|c|}{ Predicted Mass (95.4\% Credible Interval) } \\
\hline $\mathcal{U}(1,6)$ & $\mathcal{N}(-6.07,0.03)$ & $\cdots$ & $14-33 M_{\text {Jup }}$ & \multicolumn{3}{|c|}{ Minimum Compatible Age (99.7\% Lower Limit) } & $15-38 M_{\text {Jup }}$ \\
\hline$\cdots$ & $\mathcal{N}(-6.07,0.03)$ & $\operatorname{PDF}\left(M_{\text {comp }}\right)$ & \multicolumn{5}{|c|}{ Minimum Compatible Luminosity (99.7\% Lower Limit) } \\
\hline $\mathcal{U}(1,6)$ & $\cdots$ & $\operatorname{PDF}\left(M_{\text {comp }}\right)$ & $>-5.96$ dex & $>-5.95$ dex & $>-5.92$ dex & $>-5.76 \mathrm{dex}$ & $>-6.13$ dex \\
\hline
\end{tabular}

Note. Predictions from evolutionary models for various permutations of input age, luminosity, and mass distributions. Here $\mathcal{U}(a, b)$ refers to a linearly uniform distribution from $a$ to $b, \mathcal{N}(\mu, \sigma)$ is a normal distribution with a mean $\mu$ and standard deviation $\sigma$, and $\operatorname{PDF}\left(M_{\text {comp }}\right)$ is our measured probability density function for the dynamical mass of Gl $758 \mathrm{~B}$.

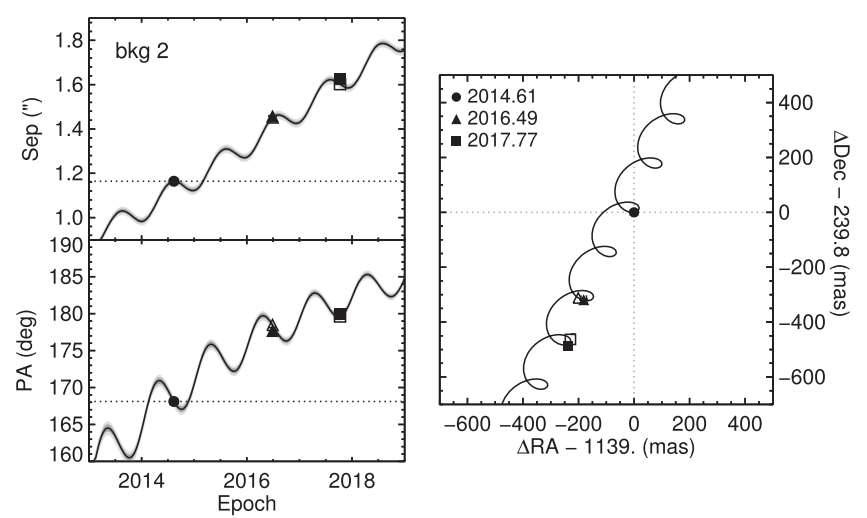

Figure 11. Astrometry of "bkg2" relative to Gl 758. This object closely follows the expected trajectory of a background star (black track with 1- $\sigma$ and 2- $\sigma$ uncertainties shown in gray) based upon the proper motion and parallax of Gl 758 in separation (top), P.A. (bottom), and relative position on the sky (right). Open symbols represent the expected position of a background object at the time of our observations; filled symbols are our measured values. Uncertainties are generally smaller than the symbol sizes. The 2014 epoch is from Vigan et al. (2016), and our new NIRC2 astrometry of bkg2 were taken in 2016 and 2017.

\& Howard (2013) measure a completeness-corrected frequency of about 55\% for all planets orbiting GK stars between 1 and $12 R_{\oplus}$ and orbital periods from 5-100 days (0.05-0.4 au), with lower-mass planets outnumbering gas giants by a factor of $\approx 50: 1$ (see also Youdin 2011; Howard et al. 2012; Fressin et al. 2013). While it is possible that the absence of close-in planets could be related to the presence of Gl $758 \mathrm{~B}$, this apparent desert is also broadly consistent with the overall statistical properties of single FGK stars.

Figure 15 shows the mass and separation regimes over which our imaging data and the residuals from our RVs are sensitive. Together this rules out giant planet and brown dwarf companions at close separations as well as massive companions at wide orbital distances. However, there exists a large region beyond about 10 au and at masses less than about $30 M_{\text {Jup }}$ where additional companions could evade detection, assuming an older age of about 6-9 Gyr. If a giant planet or another brown dwarf resides in this system-which remains possible both below our detection limits or simply at unfavorable viewing geometries - the acceleration we observe would be the superposition from one or more additional companions besides

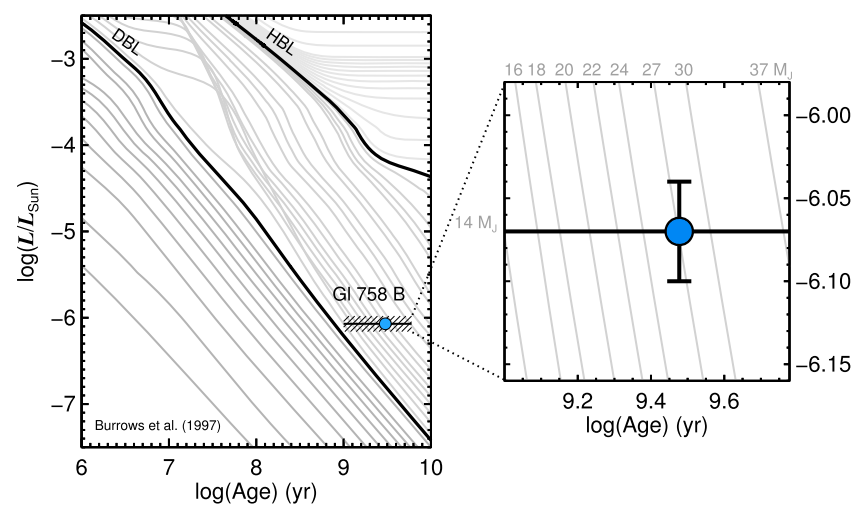

Figure 12. Luminosity and age of G1 758 B with respect to evolutionary models from Burrows et al. (1997). The inset shows iso-mass tracks spanning the nominal age range of 1-6 Gyr for the host star. The hydrogen-burning limit (HBL) and deuterium-burning limit (DBL) are labeled.

G1 758 B. This could potentially influence the amplitude and shape of the evolving acceleration and may even reconcile the dynamical mass measurement and the younger age. At this point there are no signs of another companion, but continued $\mathrm{RV}$ monitoring and deeper high-contrast imaging would be beneficial to further map the architecture of this system.

\section{Summary and Conclusion}

We have used 630 RV observations of the G8 star Gl 758 taken over the past two decades together with seven years of astrometry with NIRC2 to measure the dynamical mass of the T7-T8 brown dwarf companion G1 758 B. A joint fit of the RVs and our new astrometry with a 15-parameter Keplerian model yields a mass of $42_{-7}^{+19} M_{\text {Jup }}$ for the companion, assuming a host star prior of $0.97 \pm 0.02 M_{\odot}$, with a strict lower limit of $30.5 M_{\text {Jup }}$ and a long tail to higher masses. Gl $758 \mathrm{~B}$ orbits its host about once a century with a modest eccentricity between about 0.26 and 0.67 and a semimajor axis of 21 au. Based on our best-fit orbit solution, we expect the host star's acceleration to continue to steepen over the next several years and then reverse sign in a few decades. Despite the excellent RV precision and long observational baseline, no close-in planets are detected in the RV residuals. Based on a revised bolometric luminosity for Gl $758 \mathrm{~B}$, most evolutionary 


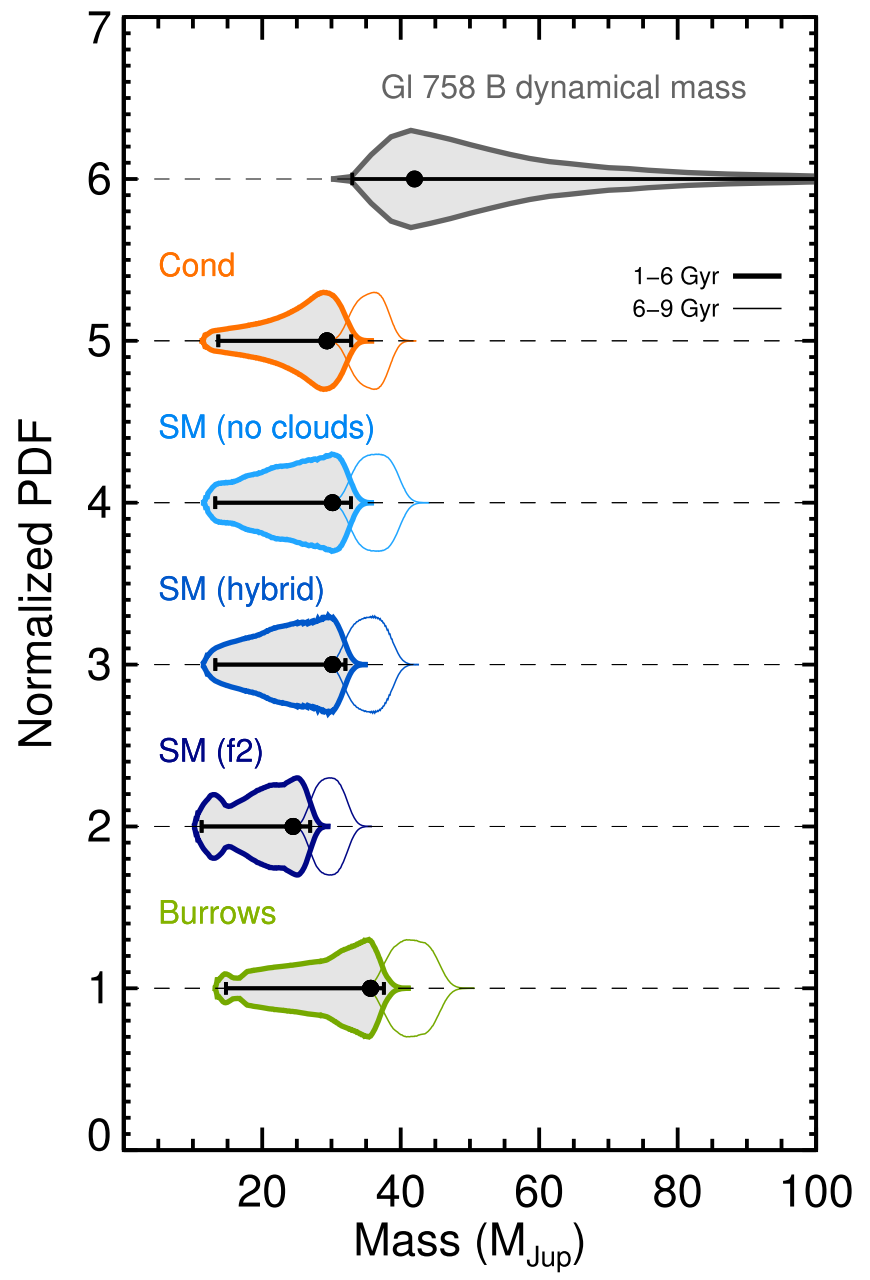

Figure 13. Probability distributions for the inferred mass of Gl 758 B from five grids of evolutionary models compared to the dynamical mass from our orbit fit (top distribution). The inferred mass distributions are calculated from the luminosity of Gl $758 \mathrm{~B}$ and the nominal age range of 1-6 Gyr for the host star (thick lines). Only the Burrows models formally agree with the dynamical mass at this age. Somewhat older ages for the host star render the models in much better agreement with the observations (thin lines).

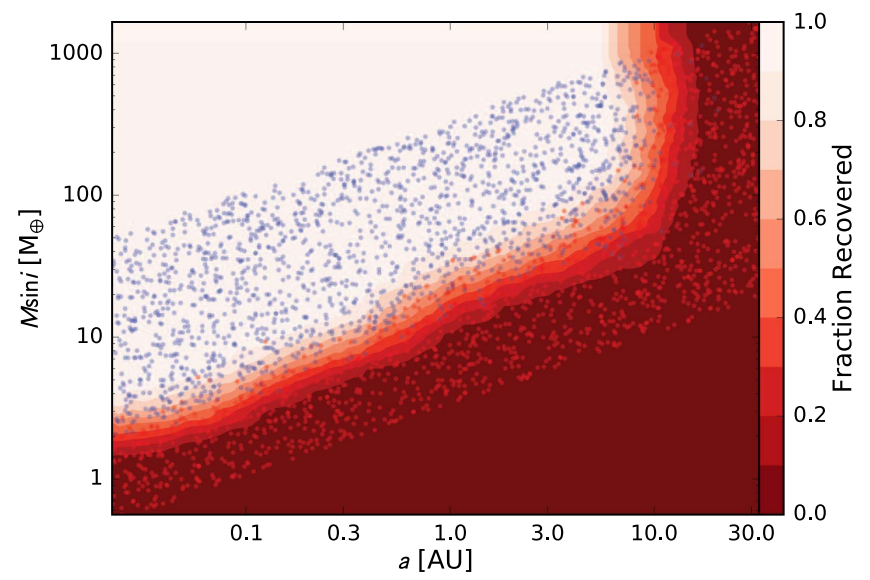

Figure 14. Sensitivity map for close-in planets orbiting Gl 758 based on residual RVs after removing the best-fit orbit for Gl $758 \mathrm{~B}$. Blue dots represent injected companions that were recovered following the procedure described in Howard \& Fulton (2016). Red dots represent injected planets that were not recovered, and contours delineate the fraction of injected planets that were recovered.

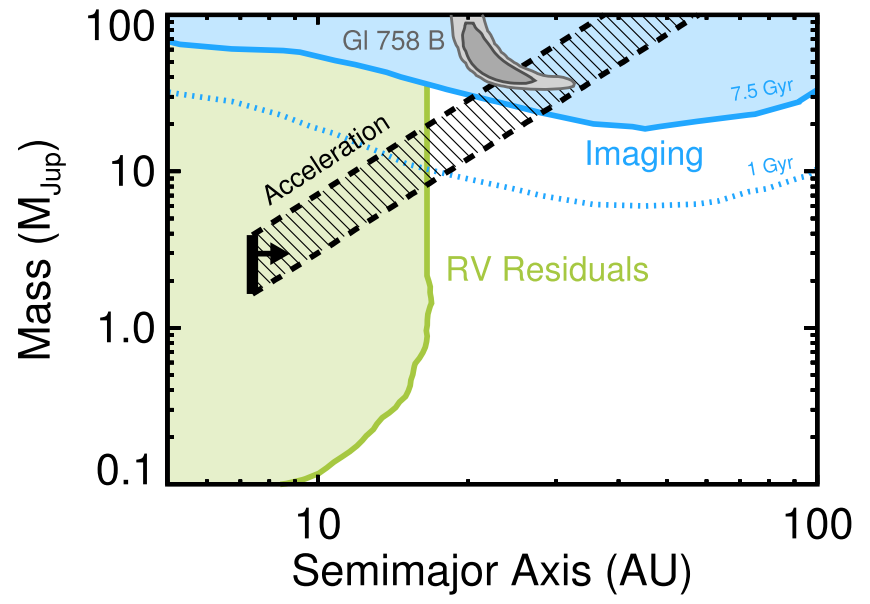

Figure 15. Overview of constraints on the G1 758 system. The shaded blue and green regions illustrate the masses and separations over which we are sensitive to companions at the $10 \%$ level based on our imaging observations and RV residuals after subtracting out the secular acceleration. The hashed area denotes the $95 \%$ interval of minimum masses and separations consistent with the measured long-baseline acceleration of $2-5 \mathrm{~m} \mathrm{~s} \mathrm{yr}^{-1}$. The cutoff at $7.5 \mathrm{au}$ corresponds to the minimum period of the perturbing companion, limited by the baseline of our McDonald RVs. Our joint constraint on the dynamical mass and semimajor axis for Gl 758 B is shown in gray, which assumes the acceleration originates entirely from this object. Note that the imaging "exclusion zone" assumes hot-start evolutionary models from Baraffe et al (2003), an age of $7.5 \pm 1.5 \mathrm{Gyr}$ for the system, and circular orbits. The corresponding contrast curve is flux calibrated using the host star and coronagraph throughput attenuation from Bowler et al. (2015a). For comparison, the dotted blue line corresponds to the $10 \%$ sensitivity contour for a younger age of $1 \mathrm{Gyr}$.

models are inconsistent with the companion's dynamical mass for ages less than $6 \mathrm{Gyr}$.

Continued ground-based RV and astrometric orbit monitoring will progressively improve the orbit and mass constraint of G1 758 B. In addition, the astrometric perturbation of Gl 758 B on its host star should be readily detected by Gaia during its nominal five year mission lifetime. This will dramatically refine the inclination and dynamical mass of Gl 758 B. Similarly, more precise age determinations for the host star will enable more robust tests of evolutionary models approaching the planetary-mass regime.

G1 $758 \mathrm{~B}$ is the lowest-mass companion inducing a measured acceleration on its host star, demonstrating the continued value and productivity of long-baseline RV planet searches. In the future, the combination of RVs and direct imaging will regularly yield dynamical masses for exoplanets using the next generation of ground-based $30 \mathrm{~m}$ class telescopes and spacebased missions like JWST and WFIRST.

We are especially grateful to the many dedicated observers and support staff at McDonald Observatory, Keck, and APF who contributed to this expansive data set over the years, in particular S. Barnes, I. Ramirez, E. Brugamyer, C. Caldwell, K. Gullikson, P. Robertson, G. Marcy, H. Isaacson, D. Fischer, K. Peek, E. Sinukoff, J. Johnson, L. Weiss, G. Torres, G. Bakos, T. Morton, J. Brewer, S. Pineda, J. Wang, C. Clanton, M. Bottom, and J. Curtis. This study would not have been possible without this communal assistance and resourcefulness. We thank Chris Sneden for many helpful discussions on the properties of the host star and Ricky Nilsson for sharing the spectrum of G1 758 B from P1640. The McDonald Observatory 
Planet Search is supported by the National Science Foundation through grant AST-1313075. This work was supported by a NASA Keck PI Data Award, administered by the NASA Exoplanet Science Institute. Data presented herein were obtained at the W. M. Keck Observatory from telescope time allocated to the National Aeronautics and Space Administration through the agency's scientific partnership with the California Institute of Technology and the University of California. The Observatory was made possible by the generous financial support of the W. M. Keck Foundation. Support for this work was provided by NASA through Hubble Fellowship grants HST-HF2-51365.001-A awarded by the Space Telescope Science Institute, which is operated by the Association of Universities for Research in Astronomy, Inc., for NASA, under contract NAS5-26555. This paper includes data taken at The McDonald Observatory of The University of Texas at Austin. The authors wish to recognize and acknowledge the very significant cultural role and reverence that the summit of Mauna Kea has always had within the indigenous Hawaiian community. We are most fortunate to have the opportunity to conduct observations from this mountain.

Facilities: Keck:II (NIRC2), Keck:I (HIRES), Smith (Tull Coudé Spectrograph), APF (Levy).

\section{ORCID iDs}

Brendan P. Bowler (1) https://orcid.org/0000-0003-2649-2288

Trent J. Dupuy (i) https://orcid.org/0000-0001-9823-1445

Michael Endl (10) https://orcid.org/0000-0002-7714-6310

William D. Cochran (i) https://orcid.org/0000-0001-

9662-3496

Benjamin J. Fulton (16 https://orcid.org/0000-0003-3504-5316

Erik A. Petigura (1) https://orcid.org/0000-0003-0967-2893

Andrew W. Howard (1) https://orcid.org/0000-0001-

8638-0320

Lea Hirsch (1) https://orcid.org/0000-0001-8058-7443

Kaitlin M. Kratter (10 https://orcid.org/0000-0001-5253-1338

Justin R. Crepp (1) https://orcid.org/0000-0003-0800-0593

Beth A. Biller (1) https://orcid.org/0000-0003-4614-7035

Marshall C. Johnson (1) https://orcid.org/0000-0002-

5099-8185

Robert A. Wittenmyer (1) https://orcid.org/0000-0001-

9957-9304

\section{References}

Artymowicz, P., \& Lubow, S. H. 1994, ApJ, 421, 651

Baraffe, I., Chabrier, G., Barman, T. S., Allard, F., \& Hauschildt, P. H. 2003, A\&A, 402, 701

Bate, M. R. 2009, MNRAS, 392, 590

Bowler, B. P. 2016, PASP, 128, 102001

Bowler, B. P., Liu, M. C., Mawet, D., et al. 2017, AJ, 153, 1

Bowler, B. P., Liu, M. C., Shkolnik, E. L., \& Tamura, M. 2015a, ApJS, 216, 7

Bowler, B. P., Shkolnik, E. L., Liu, M. C., et al. 2015b, ApJ, 806, 62

Brewer, J. M., Fischer, D. A., Valenti, J. A., \& Piskunov, N. 2016, ApJS, 225,1
Burrows, A., Hubbard, W. B., Lunine, J. I., \& Liebert, J. 2001, RvMP, 73, 719 Burrows, A., Marley, M., Hubbard, W. B., et al. 1997, ApJ, 491, 856 Campante, T. L., Barclay, T., Swift, J. J., et al. 2015, ApJ, 799, 170 Cheetham, A., Ségransan, D., Peretti, S., et al. 2017, arXiv:1712.05217 Cochran, W. D., Hatzes, A. P., \& Butler, R. P. 1997, ApJ, 483, 457 Crepp, J. R., Gonzales, E. J., Bechter, E. B., et al. 2016, ApJ, 831, 136

Crepp, J. R., Johnson, J. A., Fischer, D. A., et al. 2012, ApJ, 751, 97

Crepp, J. R., Johnson, J. A., Howard, A. W., et al. 2014, ApJ, 781, 29

Cumming, A., Butler, R. P., Marcy, G. W., et al. 2008, PASP, 120, 531

Currie, T., Bailey, V., Fabrycky, D., et al. 2010, ApJL, 721, L177

Dupuy, T. J., Kratter, K. M., Kraus, A. L., et al. 2016, ApJ, 817, 80

Dupuy, T. J., \& Liu, M. C. 2017, ApJS, 231, 0

Dupuy, T. J., Liu, M. C., \& Ireland, M. J. 2009, ApJ, 692, 729

Dupuy, T. J., Liu, M. C., \& Ireland, M. J. 2014, ApJ, 790, 133

Earl, D. J., \& Deem, M. W. 2005, PCCP, 7, 3910

Endl, M., Brugamyer, E. J., Cochran, W. D., et al. 2016, ApJ, 818, 34

Endl, M., Kürster, M., \& Els, S. 2000, A\&A, 362, 585

Foreman-Mackey, D., Hogg, D. W., Lang, D., \& Goodman, J. 2013, PASP, 125,306

Fressin, F., Torres, G., Charbonneau, D., et al. 2013, ApJ, 766, 81

Fulton, B. J., Weiss, L. M., Sinukoff, E., et al. 2015, ApJ, 805, 175

Gaia Collaboration, Brown, A. G. A., Vallenari, A., et al. 2016, A\&A, 595, A2

Howard, A. W., \& Fulton, B. J. 2016, PASP, 128, 114401

Howard, A. W., Johnson, J. A., Marcy, G. W., et al. 2010, ApJ, 721, 1467

Howard, A. W., Marcy, G. W., Bryson, S. T., et al. 2012, ApJS, 201, 15

Ireland, M. J., Kraus, A., Martinache, F., Lloyd, J. P., \& Tuthill, P. G. 2008 , ApJ, 678, 463

Janson, M., Carson, J., Thalmann, C., et al. 2011, ApJ, 728, 85

Konopacky, Q. M., Ghez, A. M., Barman, T. S., et al. 2010, ApJ, 711, 1087

Lafrenière, D., Marois, C., Doyon, R., Nadeau, D., \& Artigau, É. 2007, ApJ, 660,770

Liu, M. C., Dupuy, T. J., \& Ireland, M. J. 2008, ApJ, 689, 436

Liu, M. C., Fischer, D. A., Graham, J. R., et al. 2002, ApJ, 571, 519

Luck, R. E. 2017, AJ, 153, 1

Lucy, L. B. 2014, A\&A, 563, A126

Mamajek, E. E., \& Hillenbrand, L. A. 2008, ApJ, 687, 1264

Marcy, G. W., \& Butler, R. P. 1992, PASP, 104, 270

Marley, M. S., \& Robinson, T. D. 2015, ARA\&A, 53, 279

Marois, C., Lafrenière, D., Doyon, R., Macintosh, B., \& Nadeau, D. 2006, ApJ, 641,556

Marois, C., Macintosh, B., \& Véran, J.-P. 2010, Proc. SPIE, 7736, 77361J

McCaughrean, M. J., Close, L. M., Scholz, R.-D., et al. 2004, A\&A, 413, 1029

Nelder, J. A., \& Mead, R. 1965, CompJ, 7, 308

Nilsson, R., Veicht, A., Godfrey, P. A. G., et al. 2017, ApJ, 838, 64

Petigura, E. A., \& Howard, A. W. 2013, PNAS, 110, 19273

Potter, D., Martín, E. L., Cushing, M. C., et al. 2002, ApJL, 567, L133

Rajan, A., Rameau, J., De Rosa, R. J., et al. 2017, AJ, 154, 0

Saumon, D., \& Marley, M. S. 2008, ApJ, 689, 1327

Service, M., Lu, J. R., Campbell, R., et al. 2016, PASP, 128, 095004

Tamura, M. 2016, PJAB, 92, 45

Thalmann, C., Carson, J., Janson, M., et al. 2009, ApJL, 707, L123

Torres, G. 1999, PASP, 111, 169

Tull, R. G., MacQueen, P. J., Sneden, C., \& Lambert, D. L. 1995, PASP, 107,709

Valenti, J. A., Butler, R. P., \& Marcy, G. W. 1995, PASP, 107, 966

van Leeuwen, F. 2007, A\&A, 474, 653

Vigan, A., Bonnefoy, M., Ginski, C., et al. 2016, A\&A, 587, A55

Vogt, S. S., Allen, S. L., Bigelow, B. C., et al. 1994, Proc. SPIE, 2198, 362

Vogt, S. S., Radovan, M., Kibrick, R., et al. 2014, PASP, 126, 359

Wittenmyer, R. A., Butler, R. P., Tinney, C. G., et al. 2016, ApJ, 819, 28

Wittenmyer, R. A., Tinney, C. G., O'Toole, S. J., et al. 2011, ApJ, 727, 102

Wizinowich, P. 2013, PASP, 125, 798

Yelda, S., Lu, J. R., Ghez, A. M., et al. 2010, ApJ, 725, 331

Youdin, A. N. 2011, ApJ, 742, 38

Zechmeister, M., Kürster, M., Endl, M., et al. 2013, A\&A, 552, A78 